\title{
ISOPHOT - Photometric calibration of point sources
}

\author{
B. Schulz 1,2 , S. Huth ${ }^{2,3}$, R. J. Laureijs ${ }^{1,2}$, J. A. Acosta-Pulido ${ }^{2,3,4}$, M. Braun $2,3,7$, H. O. Castañeda ${ }^{2,3,4}$, \\ M. Cohen ${ }^{10,11}$, L. Cornwall ${ }^{2,5}$, C. Gabriel ${ }^{1,2}$, P. Hammersley ${ }^{4}$, I. Heinrichsen ${ }^{2,6,8}$, U. Klaas ${ }^{2,3}$, D. Lemke ${ }^{3}$, \\ T. Müller ${ }^{1,2,3}$, D. Osip ${ }^{9,12}$, P. Román-Fernández ${ }^{1,2}$, and C. Telesco ${ }^{9}$
}

1 ISO Data Centre, Astrophysics Division of ESA, Villafranca, PO Box 50727, 28080 Madrid, Spain

2 ISO Science Operations Centre, Astrophysics Division of ESA, Villafranca, PO Box 50727, 28080 Madrid, Spain

3 Max-Planck-Institut für Astronomie, Königstuhl 17, 69117 Heidelberg, Germany

${ }^{4}$ Instituto de Astrofisica de Canarias, 38200 La Laguna, S/C Tenerife, Spain

${ }^{5}$ Rutherford Appleton Laboratory, Chilton, Didcot, OX11 0QX, UK

6 Max-Planck-Institut für Kernphysik, Saupfercheckweg 1, 69117 Heidelberg, Germany

7 Astrophysikalisches Institut Potsdam, An der Sternwarte 16, 14482 Potsdam, Germany

8 Infrared Processing and Analysis Center, California Institute of Technology, MS 100/22, Pasadena, CA 91125, USA

9211 Bryant Space Science Center, PO Box 112055, Dpt. of Astronomy, Univ. of Florida, Gainesville, FL 32611-2055, USA

10 Radio Astronomy Laboratory, 601 Campbell Hall, University of California, Berkeley, CA 94720, USA

11 Vanguard Research, Inc. Suite 204, 5321 Scotts Valley Drive, Scotts Valley, CA 95066, USA

12 MIT, Dept. of Earth, Atmospheric and Planetary Sciences, Bldg. 54-420, 77 Massachusetts Ave., Cambridge MA 02139, USA

Received 1 August 2001 / Accepted 29 October 2001

\begin{abstract}
All observations by the aperture photometer (PHT-P) and the far-infrared (FIR) camera section (PHT-C) of ISOPHOT included reference measurements against stable internal fine calibration sources (FCS) to correct for temporal drifts in detector responsivities. The FCSs were absolutely calibrated in-orbit against stars, asteroids and planets, covering wavelengths from 3.2 to $240 \mu \mathrm{m}$. We present the calibration concept for point sources within a flux-range from $60 \mathrm{mJy}$ up to $4500 \mathrm{Jy}$ for staring and raster observations in standard configurations and discuss the requisite measurements and the uncertainties involved. In this process we correct for instrumental effects like nonlinearities, signal transients, time variable dark current, misalignments and diffraction effects. A set of formulae is developed that describes the calibration from signal level to flux densities. The scatter of 10 to $20 \%$ of the individual data points around the derived calibration relations is a measure of the consistency and typical accuracy of the calibration. The reproducibility over longer periods of time is better than $10 \%$. The calibration tables and algorithms have been implemented in the final versions of the software for offline processing and interactive analysis.
\end{abstract}

Key words. instrumentation: photometers - methods: data analysis - techniques: photometric - infrared: stars infrared: solar system

\section{Introduction}

In its broadest meaning, we define calibration as transforming the specific, nowadays digital, output of a scientific instrument to physical units. In our case these are flux densities at given wavelengths and sky positions. This transformation generally varies with every change of the instrument set-up, hence the complexity of the calibration

Send offprint requests to: B. Schulz,

e-mail: bschulz@iso.vilspa.esa.es task increases with the number of instrument configurations used. Initially it is derived on the grounds of the known instrument geometry and the relevant optical and electrical properties, that as a whole we will refer to as the ideal instrument model. Subsequently this model is refined and becomes more empirical, in order to match the measured data.

The task of calibration can be divided into three parts: First, development of the instrument model and determination of the instrument parameters that are assumed 


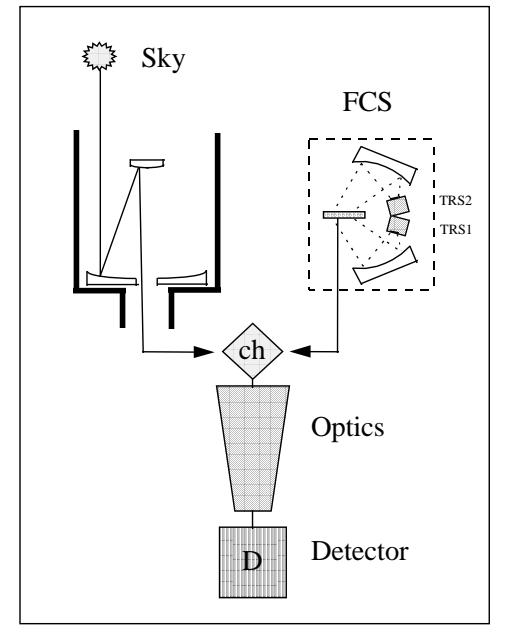

Fig. 1. Instrument schematic from a calibrator's point of view. The detector compares IR radiation directly from the sky and from the internal reference source. Stability of the detector is required only for the time interval of the two measurements.

to be unchanging and that can be measured in the laboratory, e.g. filter transmissions, aperture diameters, etc. Second, the determination of the open parameters of the ideal instrument model that can be determined only insitu, i.e. with the instrument built into the satellite and in the real space-environment. And third, the determination of deviations from the ideal instrument model that cannot be removed by adjusting parameters, and require a new functional property. These modifications to the instrument model are found empirically, and generally originate in simplifications. Because open parameters and non-ideal instrumental effects are usually intertwined, an iterative process is required to separate and quantify all the contributing effects.

This paper presents the photometric calibration of staring mode- and simple raster mode observations ${ }^{1}$ of point sources with the $\mathrm{P}$ - and C-sections of ISOPHOT (Lemke et al. 1996), which is one of four scientific instruments on board ESA's Infrared (IR) Space Observatory ISO (Kessler et al. 1996). Unlike CCD devices, the detectors for the Mid-IR (MIR) and Far-IR (FIR) are far less stable and exhibit a continuously changing relation between signal and incident flux. Thus the two Fine Calibration Sources (FCS) built into ISOPHOT played a crucial role as stable references for the photometry and most of this paper will describe their empirical calibration.

The data collected for this task represent the largest part of all specific calibration observations during the mission. This resulted in a fairly homogeneous block of data. Its analysis and comparison to modeled spectral energy distributions (SEDs), of the observed celestial standard sources yielded most of the results presented here and drove several refinements to the ideal instrument model. An additional difficulty was the large number of possible instrument configurations, which was limited somewhat by considering only one standard aperture for each filter

\footnotetext{
1 Excluding the AOT P32.
}

band of the aperture photometer (see Table 2). The set of instrument configurations and modes we treat herein defines a well-understood baseline within the large parameter space, where absolute calibration errors are expected to be minimal. The calibration of further configurations and modes, like chopped observations, extended source photometry, or non-standard apertures, is left to future publications.

We start with a brief review of the instrumental design with some emphasis on the internal reference sources (Sect. 2), followed by an outline of the calibration strategy (Sect. 3). Section 4 continues with a description of the corrections applicable to the detector signal. Section 5 presents the celestial calibrators and Sect. 6 describes those corrections imposed by photometric constraints. We derive the FCS calibration tables in Sect. 7 and present the final flux calibration, with its mathematical description and a discussion on accuracy and reproducibility, in Sect. 8. A summary constitutes Sect. 9.

\section{Instrument design}

\subsection{Optical system}

A schematic instrumental set-up is shown in Fig. 1. A more detailed description can be found in Klaas et al. (1994) or Laureijs et al. (2001). The set-up allows a choice between two sources of IR radiation via a chopper mirror: i) the telescope and ii) the internal FCS. The radiation of either source is analysed by an optical system through filters, apertures, etc., and reaches finally the IR detectors.

The telescope is a Ritchey-Chrètien design, diffractionlimited at $5 \mu \mathrm{m}$, with an entrance aperture of $60 \mathrm{~cm}$ in diameter and an $f$-ratio of 15 (Kessler et al. 1996). Radiation reaches the chopper mirror within ISOPHOT via a pyramidal mirror, which is centred between the 4 ISO-instruments. The sky is projected onto the focal plane with a scale of $0.04363 \mathrm{~mm} /{ }^{\prime \prime}$.

\subsection{Detectors}

The subsystems PHT-P and PHT-C are a multi-aperture photometer with 3 single pixel detectors and a FIR camera comprising 2 detector arrays of $3 \times 3$ and $2 \times 2$ pixels. The detectors of PHT-P are P1 (3.15-17.5 $\mu \mathrm{m})$, P2 $(15-$ $30 \mu \mathrm{m})$, and P3 $(40-130 \mu \mathrm{m})$ with 10,2 , and 2 filter bands respectively. Each detector can be combined freely with one of 11 circular field apertures with diameters between $5^{\prime \prime}$ and $180^{\prime \prime}$. Only one filter band is measured at a time. The PHT-C pixel sizes are $1.9 \times 1.9 \mathrm{~mm}$ and $3.9 \times 3.9 \mathrm{~mm}$ with gaps of $0.1 \mathrm{~mm}$ between pixels. The projected pixel sizes on the sky are $43.5^{\prime \prime} \times 43.5^{\prime \prime}$ for C100 and $89.4^{\prime \prime} \times 89.4^{\prime \prime}$ for C200.

The detectors, made from doped silicon or doped germanium, are extrinsic photoconductors operated at temperatures of 1.7 to $3.6 \mathrm{~K}$, depending on material. Their response to IR radiation is characterised by the detector responsivity, $R$, which is the parameter most relevant to 
calibration. It is given by the relation between detectorsignal and incident in-band power $R=\frac{\left(S-S_{\mathrm{dark}}\right) C_{\mathrm{int}}}{P}$, where $S$ is the detector signal expressed in $\mathrm{V} / \mathrm{s}$ for an integrating amplifier. $S_{\text {dark }}$ is the relaxed signal under dark conditions, $C_{\text {int }}$ is the integrating capacity $(140 \mathrm{fF}$ for C200, $90 \mathrm{fF}$ for others) and $P$ represents the in-band power in Watts.

The responsivity of these detectors is not constant. Depending on material, variations of a factor of 3 during one orbit were observed. Changes of $R$ are induced by the ionizing radiation in space and by the previous sequence of IR fluxes to which the detector has been exposed. We consider $R$ to be stable over typical time intervals of around 10 to $20 \mathrm{~min}$. To bring $R$ back to a nominal value, the detectors routinely underwent a curing-procedure after having crossed the Radiation Belts during perigee and before regular observations started ("science window") (see Laureijs et al. 1996, 2001). These procedures consisted of detector specific combinations of detector-heating, bias increase or illuminator flashes (Lemke at al. 1996) and brought the responsivity back to within $5 \%$ of its nominal value. The doped-Ge detectors, which showed the biggest responsivity changes, underwent a second curing procedure after 8 hours, close to apogee.

\subsection{Fine Calibration Source (FCS)}

The instrument hosts two FCSs, of which FCS 1 was used for the staring and raster observations and, hence, is chiefly treated in this paper. Both devices were manufactured at MPIA Heidelberg. Each FCS contains two thermal radiation sources (TRS) made of small diamondplates of $1 \times 1 \times 0.1 \mathrm{~mm}$, coated with $\mathrm{Ni}-\mathrm{Cr}$. The plates are heated electrically with stabilised heating powers up to $50 \mathrm{~mW}$, providing highly reproducible IR fluxes. The stabilisation circuit works digitally, with a resolution of $12.21 \mu \mathrm{W}$. This assures a flux stability of better than $4 \%$ for heating powers above $h=120 \mu \mathrm{W}$, and $2 \%$ above $h=264 \mu \mathrm{W}$.

The radiation of both sources is combined in a diamond beam-splitter. The beam of TRS 1 is reflected with practically no losses, while the beam of TRS 2 is transmitted through the material and attenuated by a factor of $\approx 1000$. Only one TRS is heated at a time. The attenuated TRS 2 is operated with the doped-Ge detectors P3, C100 and C200, which show higher responsivities, whereas the doped-Si detectors $\mathrm{P} 1$ and P2 are used with TRS 1 in the stronger reflected beam. Mirrors focus the emerging radiation in such a way that a beam with the same aspect ratio as the telescope is emitted, while the TRSs are imaged onto the chopper mirror, making the FCS appear to the detectors as a homogeneously illuminated source.

The in-band power provided by the FCS could be adjusted by changing its heating power. The fundamental calibration curves, in-band power versus FCS heating power, were initially determined by a grey body model (Schulz 1993), fitted to a few already known data points. The grey body is defined as $\epsilon B_{\nu}(T)$, which is the Planck function multiplied by a wavelength-independent emissivity $\epsilon$. The temperature, $T$, is linked to the FCS heating power, $h$, by an empirical function, $T(h)=\alpha * P^{\beta}+\gamma+$ $\delta \operatorname{atan}(P / 2)$, where $\gamma$ is the temperature of the optical support structure $(2.76 \mathrm{~K})$, and $\alpha, \beta$ and $\delta$ are dimensionless constants. Typical values for these constants of FCS $1 /$ TRS 2 are $25.8 \pm 1.8,0.518 \pm 0.006$ and $0.07 \pm 0.04$ respectively. An attenuation factor which modifies the effective solid angle individually for each detector subsystem was introduced, describing flux losses that were not predicted by the grey body model.

The advantage of the grey body model was to allow predictions for other filter bands, when only a few data points per detector were available. To increase accuracy, once enough data points were available, the covered ranges were interpolated by smooth low-order polynomials and the model was used for extrapolations only.

\section{Calibration strategy}

Given the drifting detector responsivity, the calibration had to be reestablished periodically by observing a known reference source. To avoid time-consuming slews across the sky to celestial calibration sources, we used the FCSs as an intermediate reference that could easily be compared with the telescope beam (Fig. 1). All ISOPHOT observations that were implemented as Astronomical Observing Templates (AOT) (see Klaas et al. 1994), were designed to contain at least one FCS measurement.

The absolute calibration of these secondary standards was established in-orbit by comparing their output to known celestial standards, an activity that started during the performance verification phase (PV), but continued throughout the mission due to the visibility constraints of some of the sources. To minimize errors due to potential detector nonlinearities, the FCS heating power was adjusted so that the emitted FCS flux roughly matched the flux emitted by celestial source and background. The limited linearity of the system demanded celestial calibration sources at all flux levels in all 25 filter bands.

\section{Deriving the detector signal}

To calculate the detector responsivity, $R$, we need to determine the detector signal. Ideally the signal is proportional to the detector current, exhibits only Gaussian distributed noise, and remains constant over periods of constant detector illumination. To approach this ideal in practice, a number of effects must be treated, which lead to the signal conditioning procedures described below.

\subsection{Integration ramps}

Integrating cold $(\approx 3 \mathrm{~K})$ readout electronics (CRE) (Dierickx et al. 1989) are used to amplify the currents of typically $10^{-16} \ldots 10^{-13}$ A that flow through the ISOPHOT photoconductors (Lemke et al. 1996). The photocurrent is measured from the rate at which the voltage at 


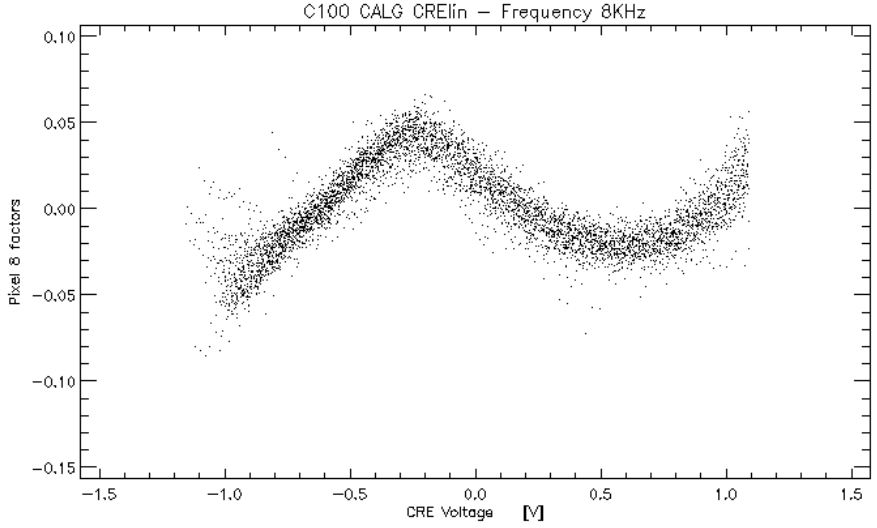

Fig. 2. Ramp nonlinearity for pixel 8 of C100. Deviations of many integration ramps from a straight line are plotted against CRE output voltage. This relation is used for ramplinearisation.

the charge capacitor within the CRE increases with time. The voltage is sampled at regular time intervals for a given duration before it is reset (non-destructive readouts, NDR, and destructive readouts, DR). The readouts of all channels of a CRE unit are time-multiplexed and sent via a single line to the external electronics unit (EEU) outside the cryostat. There they are further amplified and digitized by a 12-bit analog-to-digital-converter (ADC). The sequence of samples between two resets is called an integration ramp and ideally fits a straight line. In the following we will refer to the slope of the fitted line as "signal", measured in $\mathrm{V} / \mathrm{s}$. Multiplication by the integration capacity, for which the design value of $90 \mathrm{fF}$ (for C200 $140 \mathrm{fF}$ ) is adopted, leads to the photocurrent in Amps.

\subsection{Nonlinearities of integration ramps}

The actual integration ramps are, however, not perfectly straight (see Fig. 2) (Schulz 1993). Two effects can be separated: i) The AC-coupled CRE circuit is not a perfect integrator. The output voltage can rise only to the level of the detector bias times the small amplification of the circuit $(\approx 10)$. This saturation level is reached asymptotically via a typical RC-loading curve. High bias voltages above $10 \mathrm{~V}$ have sufficiently high saturation levels $(\approx 100 \mathrm{~V}$ and more) that they show practically linear integration ramps within the $\mathrm{CRE}$ output range of $\approx 2 \mathrm{~V}$. However, for the smaller biases of P3, C100 and C200, the saturation voltage drops such that the curvature of the loading curve appears within the dynamic range of the integration ramp (debiasing). ii) Secondly, all integration ramps show deviations from a straight line that always appear at the same CRE output voltage, regardless of the level from which the ramp started to integrate. Due to the direct link to the CRE output voltage we attribute this component to an intrinsic nonlinearity of the amplifier within the circuit.

The correction algorithm is based on the observation that all integration ramps of one detector pixel can be matched by conserving the CRE output voltage of the individual readouts, while linearly stretching and shifting the time-axis of each ramp (Schulz 1993). In this way an average integration ramp was determined for each detector pixel from a number of measurements with ramps of large dynamic range. The difference between a straightline fit to this ramp and the ramp itself (Fig. 2) is used to linearise individual ramps. The maximum absolute values range between 40 and $100 \mathrm{mV}$ over the maximum CRE dynamic range of $2 \mathrm{~V}$. The correction depends only on CRE output voltage, and has no further dependences on readout frequency, in-band power or time.

The reliability of the correction is deduced from the standard deviation of the individual ramps from the average integration ramp. Over most of the dynamic range it is below $\pm 10 \mathrm{mV}$, with a tendency towards larger scatter for longer wavelength detectors. The highest standard deviation appears in the central pixel of C100, showing $\pm 20 \mathrm{mV}$. The higher scatter towards long wavelength detectors with smaller biases is interpreted as resulting from a simplification in the correction algorithm. Relating the corrections only to the CRE output voltage neglects the fact that the curvature due to debiasing also depends on the reset level of the integration ramp, which shifts slightly w.r.t. the CRE output voltage. Hence an additional scatter appears, increasing with smaller bias. Considering the maximum standard deviation of $\pm 20 \mathrm{mV}$ and an average dynamic range of $1 \mathrm{~V}$ (half the maximum dynamic range), we estimate the typical residual systematic error to be about $\pm 2 \%$ after correction.

\section{3. lonizing radiation effects and deglitching}

The output signal is disturbed by energetic particle hits (glitches), mostly protons and electrons. A typical distribution of fitted slopes after ramp linearisation is shown in Fig. 3. The asymmetry of the distribution is caused by glitches that instantaneously increase the charge on the capacitor during the integration, so that the average slope of the affected integration ramp is increased. Typically, the readouts following the glitch continue integrating as before; however, stronger hits can affect the detector, leading to long-lasting responsivity changes. A number of algorithms have been developed to remove signals affected by glitches (Gabriel et al. 1997).

\subsection{Digitization effects}

All deglitching algorithms still leave an asymmetry in the signal distribution. For stronger asymmetries the simple average is often quite far from the peak of the distribution. The median comes closer to the peak; however, it is a bad choice for very weak signals and short integration ramps, where the bins of the $\mathrm{A} / \mathrm{D}$-conversion become significant. The AC-converter covers the range from -10 to $+9.995 \mathrm{~V}$. Dividing by the gain factor of the analog electronics and $2^{12}$ for 12 bits, the least significant bit is equivalent to $6.1 \mathrm{mV}$. For integration ramps of 1 second 


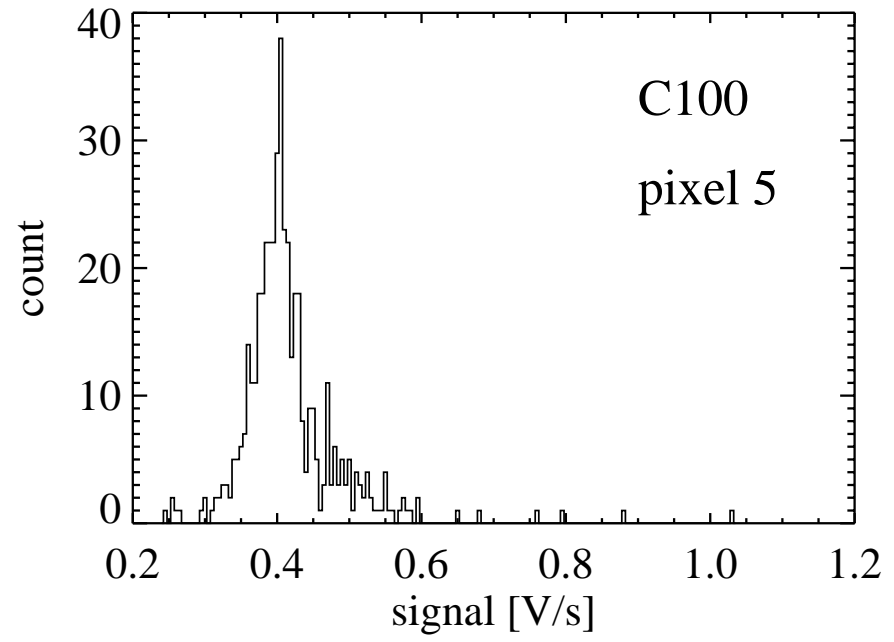

Fig. 3. Histogram showing the signal distribution in a long measurement of the central pixel of $\mathrm{C} 100$. The tail towards higher signals is caused by high energy particle hits and can be removed by appropriate algorithms.

this would yield a detector current of $5.5 \times 10^{-16} \mathrm{~A}$. As an example, the $100-\mu \mathrm{m}$ channel would produce discrete slope levels in about $20 \mathrm{mJy}$ intervals. Since the result of the median is always an existing value of the sample, the accuracy is limited to the separation of the intervals. In this case, better results can be obtained by fitting a Gaussian to the signal distribution.

\subsection{Detector transients and long-term drifts}

After a change of IR flux, the photocurrent shows a characteristic behaviour before it approaches the final value asymptotically, which we call hereafter "transient". Relaxation times of several minutes are common. The second effect, which we refer to as drift, is also observed as a gradual variation of the responsivity; however, it is on timescales of hours rather than minutes. Drift is due to the continuous bombardment of the detector material by high energy particles in space (e.g. see Blum et al. 1990), but can also be triggered by strong flux changes. In the following we describe in more detail how the transient correction was applied to the FCS calibration data, since this was an important step in eventually achieving consistent photometric results.

\subsubsection{Solutions for transients}

The observed detector current is the sum of dark current and a current proportional to the number of incident IR photons (Bratt 1977; Sclar 1984). However, this is correct only for a system that has reached equilibrium after each flux change. To derive signals that are independent of measurement time, we need to determine the signal as $t \rightarrow \infty$. The simplest solution is to determine the level when the signal has practically reached its final value and no trend is detected within the noise. To filter out the cases that need special treatment, a "Stability Check", implemented in the PHT Interactive Analysis (PIA) software (Gabriel et al. 1997), is conducted. The method tests successively smaller pieces of data towards the end of a measurement for stability at a $95 \%$ confidence level. The smallest piece is limited to either 7 data points or $8 \mathrm{~s}$. Stable signal levels were detected for P1, P2 and C100 for about $65 \%$ of our calibration measurements. Typical measurement times were between 64 and $256 \mathrm{~s}$. For fainter signals, however, the relaxation times generally increase and other methods had to be applied to predict the final signal level from only the first part of the transient.

\subsubsection{Empirical transient fit}

Transients converge asymptotically towards a final value, $S_{\infty}$. Empirical functions that fit this characteristic are usually constructed from exponentials. Since long term detector drifts bound the integration time above, typical durations of "staring" measurements at one flux level range between 64 and $256 \mathrm{~s}$. We use an empirical function of the form $S(t)=S_{\infty}+\left(S_{0}-S_{\infty}\right) \mathrm{e}^{-c t} . S_{0}$ is the signal before the flux change, $t$ is time and $c$ is a free parameter. Since in most cases $S_{0}$ is not known, it is also left as a free parameter to fit. Stronger transients that are "remembered" by the detector during successive measurements can be eliminated by fitting a "baseline" of the same expression, if more than one measurement of the same flux level is obtained (see Fig. 4).

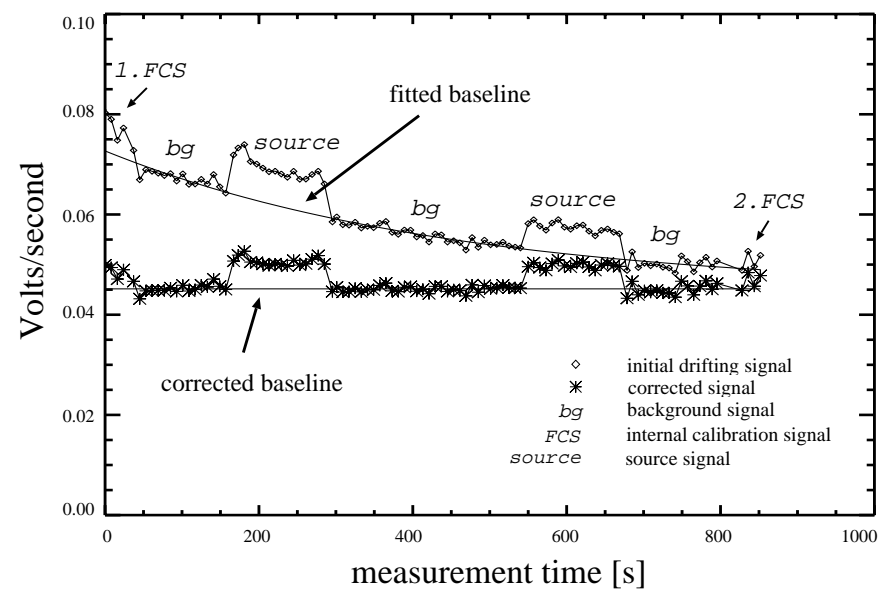

Fig. 4. Observations in "nodding mode" with a linear 3-step raster used for faint sources. Several changes of the pointing between a strong background and a faint source on top of it make the source signal appear as a modulation on top of a strong transient. This is corrected by dividing by a baseline fitted to all background levels. The sequence starts and ends with FCS measurements.

\subsubsection{Automatic validation of transient fits}

Automatic procedures were developed to reduce the calibration data homogeneously. The routines include stability test, empirical transient fit and error 
discrimination $^{2}$ (Huth \& Schulz 1998). If the stability test cannot locate a stable part of the measurement within the criteria given above, a transient fit is initiated. The criteria that were developed during intensive tests to eliminate dubious results from transient fitting are as follows:

(i) We calculate the average of the last $30 \%$ of the measurement and call it $S_{30}$. The valid fit result must deviate by no more than $\pm 50 \%$ from $S_{30}$.

(ii) A straight line is fitted to the last $40 \%$ of the measurement and extrapolated to a point at 2 times the measurement time. $80 \%$ of the difference between this point and $S_{30}$ is the maximum difference allowed between the signal level predicted by the transient fit and $S_{30}$.

(iii) For each fitted transient, the term $\left(1-S_{1} / S_{2}\right) /$ $\left(t_{1}-t_{2}\right)$ is evaluated twice, where $S$ and $t$ are the slopes and times of either the first two, or the last two, data points in the measurement. Absolute differences between these two results that are smaller than $0.001 \mathrm{~s}^{-1}$ are rejected. This and criterion (ii) buffer the curvature of the fit against small values since small curvatures result in very uncertain asymptotes.

In case these criteria fail, we take $S_{30}$ as the best guess for $S_{\infty}$ but the result is flagged as being less reliable. This applied to about half the cases where transient fits were attempted. We caution that the criteria were tuned empirically to this large, but special, sample of FCS calibration measurements. We assume, therefore, that they work best for measurement times $\geq 64$ s.

\subsubsection{Signal uncertainties}

In the automatic processing we distinguish three types of measurement. The first consists of measurements that reach stability within their integration time. Here the statistical error is the standard deviation of the mean of the stable part of the measurement. The second type consists of transient fitted measurements that meet the criteria above, and we use $\sqrt{1 /(n-1) \sum_{i}^{n}\left(S_{i}^{\text {modl }}-S_{i}^{\text {meas }}\right)}$ as error, where $n$ is the number of valid slopes in the measurement, and $S^{\text {modl }}$ and $S^{\text {meas }}$ are the modeled and measured slopes respectively. If the transient function were a constant, i.e. a horizontal line, the expression would be the standard deviation. We find that using the above equation, instead of the standard deviation of the mean, provides more realistic errors. Finally, in cases where no stability is found and the transient fit fails, the error assigned is the standard deviation of the last $30 \%$ of the measurement. Thus the errors of the three types also weight the results with respect to their systematic uncertainties.

\footnotetext{
2 Note that the quality check function was specifically developed to process FCS calibration measurements automatically. Although this check is not available in the PIA software, the described transient correction can be interactively applied by the user, so that our conclusions are not necessarily limited to our specific set of measurements only.
}

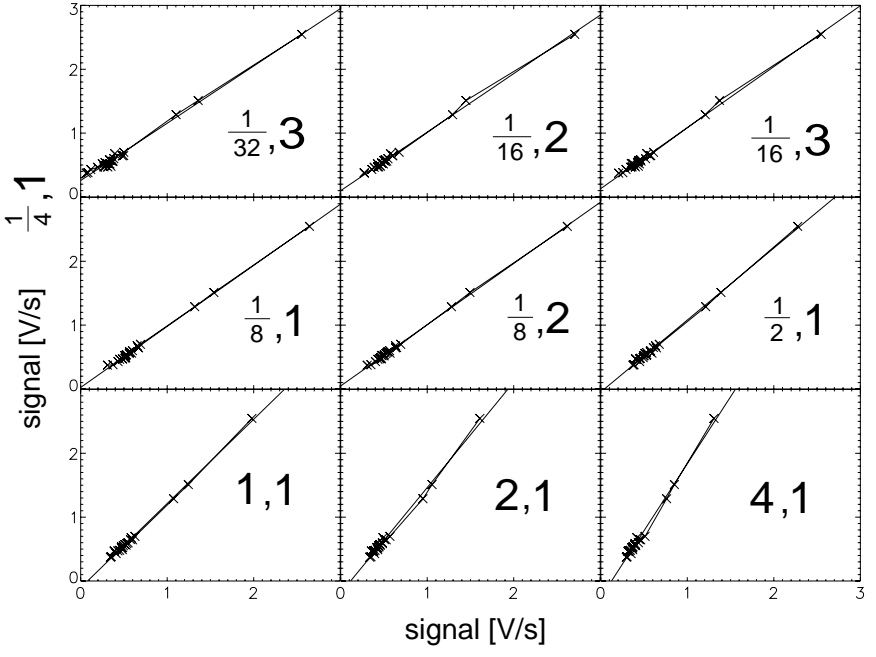

Fig. 5. The diagram shows the relations of signals of the same in-band power measured with different readout timing settings. The signals on the $y$-axis are measured using a $1 / 4$ s reset interval and a data reduction factor of 1 . The reset interval and data reduction factor, respectively, for the signals of the $x$-axis are given by the two numbers in each diagram. The data points follow a linear relation, with slope different from unity and non-zero $x$-intercept. This example was measured for detector C100.

\subsection{Signal dependence on readout timing}

The readout timing ( $\mathrm{ROT}$ ) of the $\mathrm{CRE}$ was selected individually for each measurement according to the observer's flux estimate, to give a maximum dynamic range without saturation and thus a minimum of readout and samplingnoise. It is controlled by 3 parameters: the time interval between two destructive readouts, $t_{\text {reset; }}$ the number of non-destructive readouts, $n_{\mathrm{NDR}}$; and the data reduction factor. For the range of $128 \mathrm{~s}$ to $1 / 64 \mathrm{~s}$ for $t_{\text {reset }}$, depending on the detector, a total of 14 to 15 combinations was actually used during operations.

We observed several non-variable ${ }^{3}$ celestial sources (stars, planets) with all ROT combinations suitable for inband powers that cover a range of a factor of 16 above and below that actually observed. Care was taken to ensure the stability of the detector response during the measurement series by repeating the set of ROT combinations in reverse order. Ideally one expects to find the same signal within the errors for the same source, regardless of CRE set-up. However, even after correcting for ramp nonlinearity, we derived different signals for the same source. The signals show a strong dependence on the reset interval, especially for those cases where part of the integration ramp exceeds the dynamic range and is discarded due to saturation.

To achieve consistency, we correct all signals so they appear as if measured with a common reset interval of $t_{\text {reset }}=1 / 4 \mathrm{~s}$ and a data reduction factor of 1 . The signal, $S$, is corrected according to $S^{\prime}=A 0+A 1 \times S$, where the parameters $A 0$ and $A 1$ depend on the ROT setup. They were determined from a least-squares fit to the

\footnotetext{
${ }^{3}$ For the time of the observation.
} 

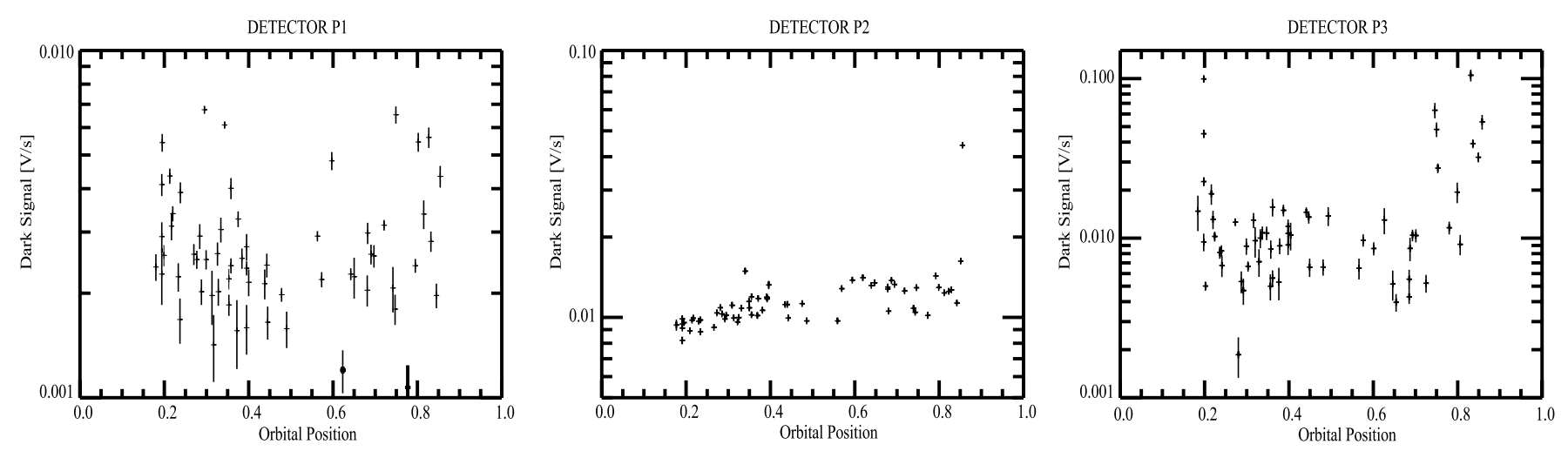

Fig. 6. The evolution of the dark signal with relative orbital position. The data points were taken randomly during the mission and plotted versus orbital position, where the range 0 to 1 corresponds to the full 24 hour orbit, starting at perigee.
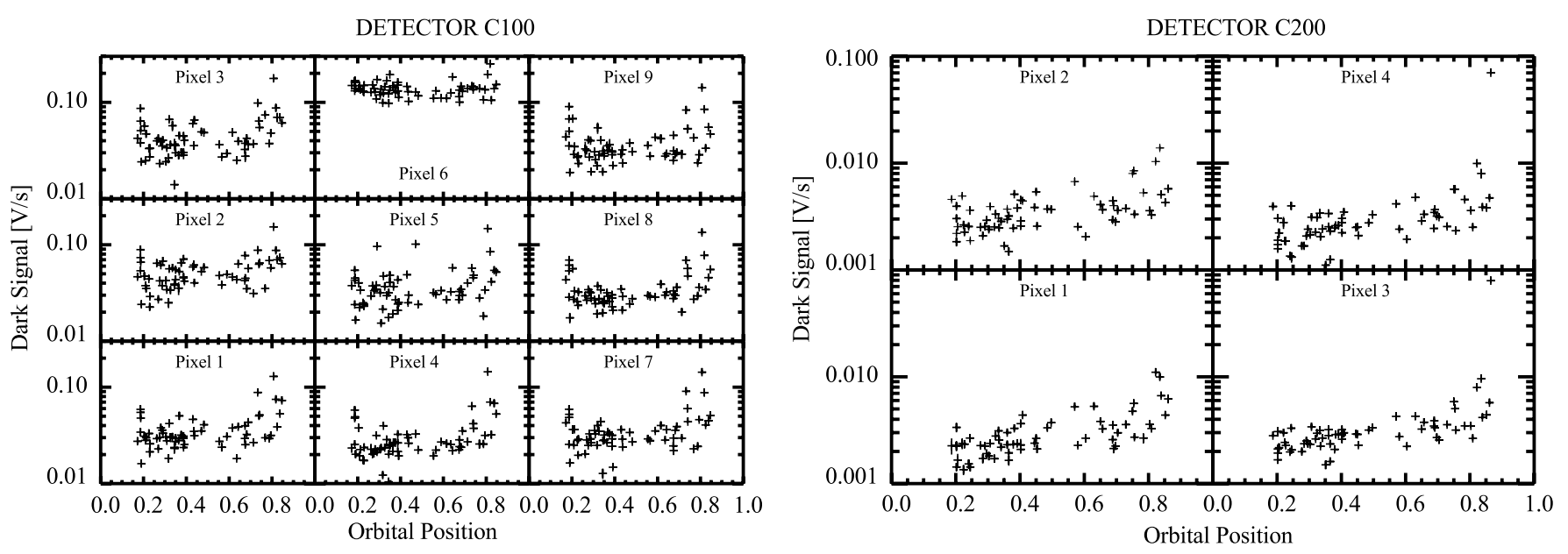

Fig. 7. As for Fig. 6 but for the detectors C100 and C200.

calibration data. An example is shown in Fig. 5. It should be noted that $A 0$ is generally different from zero and the slope $A 1$ is significantly different from unity. All pixels of equal detector-subsystems were found to follow the same relation, suggesting that a pure CRE-effect is observed. Again the residual errors of the correction are systematic in nature. The scatter of the calibration data around the fits suggests typical systematic errors of no more than $5 \%$ and probably better than $2 \%$.

\subsection{Dark current}

Even under completely dark conditions and after relaxation of transients due to previous illumination, a detector current is measured, arising from thermal generation of free charge carriers and surface leakage conductance (Bratt 1977). This value must be subtracted to obtain the pure photocurrent. The dark current had already been determined for each detector pixel in the laboratory; however, in orbit higher dark currents than on the ground were generally observed. A comparison is given by Lemke et al. (1996). We interpret part of the increase as due to the faint end of the glitch distribution, where many small glitches produce a continuous current (see Fig. 3). Dark signals measured randomly over the mission versus relative orbital position are plotted in Figs. 6 and 7 . The range 0 to 1 on the $x$-axis corresponds to the full 24-hour revolution, starting at perigee. The data show a dependence of the dark signal on orbital position. The systematic rise along the orbit is attributed to high energy particles in space. In particular, the steep rise at the end indicates the beginning of entry into the Radiation Belts. The uncertainty in the dark signals is due to detector noise and limitations in correcting detector transients. A low order polynomial fit to these diagrams is used to model the time-dependence of the dark current over the orbit.

\section{Celestial calibrators}

The absolute photometric calibration of ISOPHOT is based entirely on point sources, which constitute the best known IR flux densities in the sky. The sources were observed either for PHT-P at the centre of a standard circular aperture that is defined per filter ${ }^{4}$ (see Table 2) or,

\footnotetext{
4 The standard apertures were chosen to include at least $90 \%$ of the energy of the point-spread-function (PSF).
} 
for the PHT-C array detectors, at the centre of each individual pixel. As the detailed radiation spectrum of the FCS was unknown and linearity of the detectors could not a priori be postulated, the empirical FCS calibration required the availability of calibration standards covering the full observable flux range between 3 and $240 \mu \mathrm{m}$. The upper limit of the absolute flux range is determined by the dynamic range of the CRE and the detector responsivity. The lowest signals are mainly determined by the celestial background and the detector responsivity.

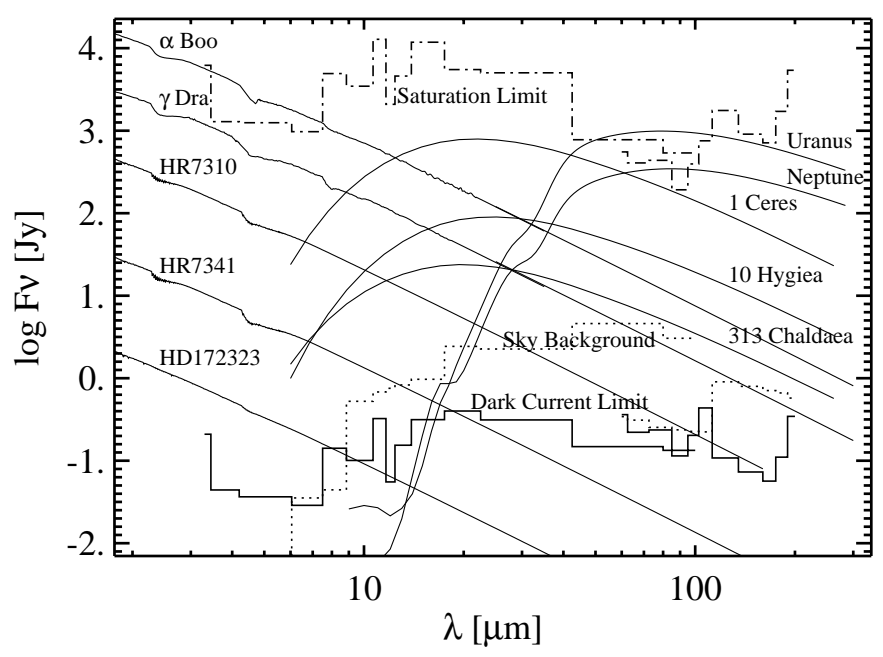

Fig. 8. The coverage of the flux domain accessible to ISOPHOT by the three kinds of celestial standard.

In Fig. 8 we illustrate the coverage of the flux domain accessible to ISOPHOT by celestial standard sources. The largest background components are zodiacal thermal reemission, peaking around $20 \mu \mathrm{m}$, and Galactic cirrus, contributing most at the longest wavelengths. Only at the shortest wavelengths does the dark current dictate the lowest signals. The dotted line shows typical surface brightnesses for celestial backgrounds, converted to an equivalent flux density of a point source, observed in the standard aperture without background. The upper absolute flux limits are plotted as dashed lines. Note that the limiting sky background for the overlapping wavelengths of PHT-P and PHT-C is different due to their different aperture areas. Examples of each of the three types of calibration source (planets, asteroids, stars) are plotted as solid lines.

\subsection{Stars}

A large database of SEDs of standard stars was prepared in support of the ISO calibration. Important aspects of the source selection were non-variability, no known stellar companions, no IR excess, low sky background and good visibility by ISO throughout the mission.

The ISO Ground Based Preparatory Programme (GBPP) (Jourdain de Muizon \& Habing 1992; van der Bliek et al. 1992; Hammersley et al. 1998) provided SEDs between 1 and $160 \mu \mathrm{m}$ by interpolating in the Kurucz stellar model grids (Kurucz 1993), using temperature, surface gravity and metallicity. The temperatures were derived either from the IR Flux Method (Blackwell et al. 1991, 1998 ) or the $V-K$ versus $T_{\text {eff }}$ relationship (Di Benedetto $1993,1998)$. Surface gravities and metallicities were from Cayrel de Strobel et al. (1992). The spectral shapes derived from the grids were then normalized using near-IR (NIR) photometry ( $K n$ or $K$-band). The attributed errors range between $3 \%$ and $5 \%$ which is dominated by the error in conversion to an absolute flux density scale. The random scatter between the measured and predicted fluxes at $10 \mu \mathrm{m}$ is shown to be better than $1.5 \%$ (Hammersley et al. 1998).

Another calibration programme by Cohen et al. (1996) provided empirical SEDs in the range from 1.2 to $35 \mu \mathrm{m}$ by splicing together measured spectral fragments of cool K- and M-giants and calibrating them by absolutely calibrated Kurucz models of Sirius and Vega. The measured "composite" SEDs are used to derive so-called "template" SEDs for fainter stars (Cohen et al. 1999), assuming that the intrinsic spectral shape depends only on spectral type and luminosity class. The absolute flux level is set by well-characterised NIR and MIR photometry, including IRAS data. To extend those composite-SEDs not observed as far as $35 \mu \mathrm{m}$ by the Kuiper Airborne Observatory, Engelke functions (Engelke 1992; Cohen et al. 1995, 1996), with effective temperatures from Blackwell et al. (1991), were used. To create FIR extensions to support the longest wavelengths of ISOPHOT $(240 \mu \mathrm{m})$, continuum model atmospheric spectra were attached to the empirical composites and extended to $300 \mu \mathrm{m}$ (Cohen at al. 1996). The typical absolute accuracy (i.e., in $F_{\lambda}$ or $F_{\nu}$ at any wavelength) of the templates is about $3 \%$, while the FIR extensions ranging from 25 to $300 \mu \mathrm{m}$ have computed uncertainties of about $6 \%$.

Note that both programmes deliberately use the same Vega model SED for their zero point definitions. Furthermore, the measured zero points of the various photometric systems were determined to ensure that there would be no systematic differences depending upon which data were used (Cohen et al. 1999). Specifically for ISO, both programmes supplied electronic versions of their SEDs with spectral resolutions of typically 50 to 300 . The objects are listed in Table 1.

The irradiances of a number of primary (Sirius, Vega), secondary (bright K- and M-giants) and tertiary (template-SEDs) calibration stars have been absolutely validated by a dedicated radiometric calibration experiment carried out on the US Midcourse Space Experiment (MSX: Mill et al. 1994) by Cohen et al. (2001). The $1.2-35 \mu \mathrm{m}$ spectra of seven of the stars in Table 1 have been validated by this means. We note that because of its observed deviation from the model SED longward of about $17 \mu \mathrm{m}$, we do not use observations of Vega to calibrate any long wavelength filter.

Brighter flux levels in the FIR (i.e. filters with reference wavelengths $\geq 50 \mu \mathrm{m}$ ) had to be calibrated by other 
Table 1. The list of stars, planets and asteroids used for ISOPHOT calibration. The flux densities $F_{\nu}$ are given at the reference wavelengths of the filters P_11.5 and C_60, depending if the respective standard was used in the range 3-30 $\mu \mathrm{m}$ or $45-240 \mu \mathrm{m}$. In addition we list the magnitudes in the ISOPHOT photometric system with the Vega model SED as zero point. The identifiers MC for M. Cohen and PH for P. Hammersley indicate the SED version that was actually used for calibration. Asterisks indicate absolute validation by MSX (Cohen et al. 2001). For solar system objects we give the brightness range observable over the ISO mission.

\begin{tabular}{|c|c|c|c|c|c|c|c|c|c|c|}
\hline \multicolumn{8}{|c|}{ Stellar Celestial Standards } & \multicolumn{3}{|c|}{ Solar System Standards } \\
\hline Name & $\begin{array}{c}\text { Common } \\
\text { Name }\end{array}$ & Src. & $\begin{array}{l}\text { Spec. } \\
\text { Type }\end{array}$ & $\begin{array}{c}F_{\nu}^{12 \mu \mathrm{m}} \\
\mathrm{Jy} \\
\end{array}$ & $\begin{array}{c}\text { (P_11.5) } \\
\text { mag }\end{array}$ & $\begin{array}{c}F_{\nu}^{60 \mu \mathrm{m}} \\
\mathrm{Jy} \\
\end{array}$ & $\begin{array}{c}\text { (C_60) } \\
\text { mag }\end{array}$ & Name & $\begin{array}{c}F_{\nu}^{60 \mu \mathrm{m}} \\
\mathrm{Jy}\end{array}$ & $\begin{array}{c}\text { (C_60) } \\
\text { mag }\end{array}$ \\
\hline HD172323 & - & $\mathrm{PH}$ & F9V & 0.065 & 6.55 & & & Uranus & $913 \ldots 972$ & $-7.28 \ldots-7.21$ \\
\hline HD184400 & - & $\mathrm{PH}$ & F5 & 0.027 & 7.53 & & & Neptune & $301 \ldots 317$ & $-6.07 \ldots-6.01$ \\
\hline HR337* & $\beta$ And & $\mathrm{MC}$ & MOIIIv & 192 & -2.05 & 7.97 & -2.17 & 1 Ceres & $248 \ldots 433$ & $-6.46 \ldots-5.85$ \\
\hline $\mathrm{HR} 617^{*}$ & $\alpha$ Ari & $\mathrm{MC}$ & K2III & 55.7 & -0.76 & 2.33 & -0.83 & 2 Pallas & $55.3 \ldots 208$ & $-5.67 \ldots-4.23$ \\
\hline HR $1457^{*}$ & $\alpha \mathrm{Tau}$ & $\mathrm{MC}$ & K5III & & & 19.1 & -3.12 & 3 Juno & $37.6 \ldots 62.2$ & $-4.36 \ldots-3.81$ \\
\hline HR1654 & $\epsilon$ Lep & $\mathrm{MC}$ & K5IIIv & & & 1.61 & -0.43 & 4 Vesta & $153 \ldots 291$ & $-6.03 \ldots-5.34$ \\
\hline HR3748 & $\alpha$ Hya & $\mathrm{MC}$ & K3II-III & 93.4 & -1.31 & 3.92 & -1.39 & 10 Hygiea & $29.0 \ldots 43.9$ & $-3.97 \ldots-3.52$ \\
\hline HR5340* & $\alpha$ Boo & $\mathrm{MC}$ & K1III & 506 & -3.15 & 21.0 & -3.22 & 54 Alexandra & $20.0 \ldots 26.1$ & $-3.42 \ldots-3.13$ \\
\hline HR5886 & - & $\mathrm{PH}$ & A2IV & 0.251 & 5.09 & & & 65 Cybele & $13.4 \ldots 22.3$ & $-3.24 \ldots-2.69$ \\
\hline HR5986 & - & $\mathrm{PH}$ & F8IV-V & 2.24 & 2.71 & & & 106 Dione & $3.80 \ldots 3.93$ & $-1.35 \ldots-1.32$ \\
\hline HR6514 & - & $\mathrm{PH}$ & $\mathrm{A} 4 \mathrm{~V}$ & 0.095 & 6.14 & & & 313 Chaldaea & $7.74 \ldots 8.14$ & $-2.15 \ldots-2.10$ \\
\hline HR6688 & - & $\mathrm{PH}$ & K2III & & & 0.463 & 0.92 & 532 Herculina & $16.3 \ldots 44.5$ & $-3.99 \ldots-2.91$ \\
\hline HR6705* & $\gamma$ Dra & $\mathrm{MC}$ & K5III & 108 & -1.46 & 4.55 & -1.56 & & & \\
\hline HR6847 & - & $\mathrm{MC}$ & G2V & 0.337 & 4.76 & & & & & \\
\hline HR7001* & $\alpha \operatorname{Lyr}$ & $\mathrm{MC}$ & $\mathrm{A} 0 \mathrm{~V}$ & 27.0 & 0.00 & & & & & \\
\hline HR7310 & - & $\mathrm{MC}$ & G9III & 14.5 & 0.67 & 0.586 & 0.67 & & & \\
\hline HR7341 & - & $\mathrm{PH}$ & K1III & 0.951 & 3.64 & & & & & \\
\hline HR7451 & - & $\mathrm{PH}$ & F7V & 0.427 & 4.51 & & & & & \\
\hline HR7469 & - & $\mathrm{PH}$ & F4V & 1.07 & 3.51 & & & & & \\
\hline HR7633 & - & $\mathrm{PH}$ & K5II-III & 9.66 & 1.14 & 0.391 & 1.11 & & & \\
\hline HR7742 & - & $\mathrm{PH}$ & K5III & 3.66 & 2.18 & & & & & \\
\hline HR7980 & $\omega$ Cap & $\mathrm{MC}$ & M0III & & & 1.17 & -0.08 & & & \\
\hline HR8684 & - & $\mathrm{PH}$ & G8III & 8.45 & 1.27 & & & & & \\
\hline HR8775* & $\beta \mathrm{Peg}$ & $\mathrm{MC}$ & M2.5II-I & 265 & -2.44 & 11.2 & -2.54 & & & \\
\hline
\end{tabular}

objects, since even the brightest standard star measured by ISOPHOT ( $\alpha$ Boo) drops to less than 2 Jy at $200 \mu \mathrm{m}$ (see Fig. 8).

\subsection{Planets}

Uranus and Neptune are commonly used as submillimeter and FIR standards (Hildebrand et al. 1985; Orton et al. 1986; Griffin \& Orton 1993). They provided the highest calibrated flux levels that ISOPHOT could measure at long wavelengths. Mars was already too bright when it became visible to ISO.

In an effort to homogenize the calibration of ISOPHOT and the Long Wavelength Spectrometer (LWS) in the long wavelength range, for the final version of the offline processing software (OLP 10) we changed the planet models used in earlier versions (Schulz et al. 1999). The ones supplied by Abbas (1997, priv. comm.) were replaced by models produced by Griffin \& Orton for LWS. The models are calibrated with 0.35 to $3.333 \mathrm{~mm}$ JCMT data (Griffin \& Orton 1993), which in turn were calibrated against Mars (Wright 1976; Wright \& Odenwald 1980). At the short wavelength end, the model was constrained by Voyager IRIS data from 25 to $50 \mu \mathrm{m}$ by Hanel et al. (1986) for
Uranus, and Conrath et al. (1989) for Neptune. The temperature structure and composition of $\mathrm{H}_{2}, \mathrm{He}$ and $\mathrm{CH}_{4}$ was taken from Voyager radio occultation experiments by Lindal et al. (1987) for Uranus, and by Lindal (1992) for Neptune.

We compared the models of Griffin \& Orton (GO) with the ones of Abbas (AB). Above $45 \mu \mathrm{m}$ the $\mathrm{GO}$ model of Neptune is $\approx 10 \%$ brighter than $\mathrm{AB}$. In contrast, for Uranus the GO model fluxes are about 3 to $10 \%$ lower than $\mathrm{AB}$ above $50 \mu \mathrm{m}$ and deviate no more than $15 \%$ above $20 \mu \mathrm{m}$. At shorter wavelengths the discrepancies between $\mathrm{AB}$ and $\mathrm{GO}$ rise dramatically for both planets. However, these are no longer relevant to the PHT calibration, since the C_50 filter passband starts only around $40 \mu \mathrm{m}$. We take these differences as indicative of how well the fluxes from Uranus and Neptune are known at FIR wavelengths and conclude that, for our purposes, they are still within a $\pm 10 \%$ margin.

\subsection{Asteroids}

A small group of asteroids was chosen to be used as FIR calibration standards. They populate the intermediate-flux level gap that appears at wavelengths 
from 45 to $200 \mu \mathrm{m}$ between Uranus, Neptune and the brightest stars (see Fig. 8).

Some specific observational complications arose, however, since they are moving objects with respect to the sky background and show periodic variations of intensity due to rotation and varying distance from both the Earth and Sun. Selection criteria were: well understood rotational behaviour, small lightcurve amplitude, some form of independent size determination (either direct imaging or via occultation measurements), good visibility during the ISO mission, and availability of sufficient observational data from visible to submillimetre wavelengths. The selection resulted from a combination of extensive groundbased observing campaigns at thermal wavelengths from the IRTF, UKIRT, and JCMT, additional visible wavelength lightcurve measurements, and a series of FIR observations from the now retired KAO. These observations were used to confirm the validity of the subsequent thermophysical modeling effort.

Initially, modified versions of the Standard Thermal Model (Lebofsky 1989) were used. For the final ISOPHOT photometric calibration, a thermophysical model (TPM) assuming a rotating ellipsoid and parameterising heat conduction, surface roughness, and scattering in the regolith was adopted for 10 asteroids (Müller \& Lagerros 1998). The TPM is capable of producing thermal lightcurves and spectral energy distributions for any time, taking into account the real observing and illumination geometry. The overall comparison between model predictions and our large sample of MIR, FIR, and submillimetre observations (about 700 individual measurements between 2 and $2000 \mu \mathrm{m})$ demonstrated an accuracy of approximately $\pm 10 \%$ across a wide wavelength range from 10 $500 \mu \mathrm{m}$. In a few cases, existing direct measurements of shape significantly improved the model accuracies.

In a recent study of the accuracy of the TPM, ISOPHOT observations of asteroids were independently calibrated in the FIR (Müller \& Lagerros 2001, 2001a). In this work, parts of the FCS power curves were established using measurements only of planets or stars. These were used then to calibrate the asteroid observations and compare the results to the TPM predictions. It was shown that observations and predictions for 1 Ceres, 2 Pallas and 4 Vesta agree within 5\%. For 3 Juno, 10 Hygiea, 54 Alexandra, 65 Cybele, and 532 Herculina the agreement is still within 10 to $15 \%$. For the 2 objects without independent ISO data the comparison to ground based thermal observations gave rms values of $14 \%$ for 313 Chaldaea and $29 \%$ for 106 Dione. Note that the rms values include also observational errors, which are dominated by the structured FIR sky background and calibration uncertainties. Modeling limitations are mainly due to uncertainties about the exact asteroidal shapes. The final modeling results were also confirmed to an accuracy of better than $10 \%$ via comparison with rotationally resolved target observations from the IRAS database. Table 1 lists the solar system objects that were used for ISOPHOT calibration.

\subsection{In-band power calculation}

To determine the responsivity of a detector we need to calculate the in-band power. Given the power distribution of a celestial standard in the focal plane, i.e. the normalized point spread function ${ }^{5}, P S F(x, y, \lambda)$, times the flux density of the source, $F_{\lambda}(\lambda)=\left(c / \lambda^{2}\right) F_{\nu}$, we define the inband power as the integral over the product of i) the flux density of the source, ii) the PSF, iii) all spectral transmissions $T_{\mathrm{f}}(\lambda)$ and iv) the relative spectral response function of the detector $R_{\mathrm{p}}(\lambda)$, normalized to unity at its peak response. The integration is performed over all wavelengths and over the area of the detector aperture.

$P_{\mathrm{src}}=T_{\mathrm{r}} \int\left[T_{\mathrm{f}}(\lambda) R_{\mathrm{p}}(\lambda) F_{\lambda}(\lambda) \iint P S F(x, y, \lambda) \mathrm{d} x \mathrm{~d} y\right] \mathrm{d} \lambda$.

Wavelength-independent losses due to mirrors are accounted for by the factor $T_{\mathrm{r}}$. The indices "p" and "f" indicate dependence on pixel or filter, respectively.

The filter transmissions (including the out-of-band rejections over a wide wavelength range) and the relative response functions of the detectors were measured in the laboratory under "cold" conditions (at their anticipated operating temperatures in-orbit) with a Fourier-transform spectrometer (Schubert 1993). To calculate $T_{\mathrm{r}}$, we assumed the reflectance of each mirror surface to be $98 \%$ and wavelength-independent. Thus we obtain $T_{\mathrm{r}}=0.98^{n}$, with $n$ being the number of mirrors in the optical paths, which is $6,7,7,6$ and 5 for the detectors P1, P2, P3, C100 and C200, respectively. For P1 an additional multiplicative factor of 0.95 was included to account for losses due to the Fabry lens.

We note that the in-band power is only a fraction of the total incident IR power, since the formula includes the wavelength-dependent relative response function of the detector material. Normalizing this response function to 1.0 at its peak allows us to define the detector responsivity as characteristic of the detector pixel, independent of the filter used. Thus $P_{\mathrm{src}}$ represents only the fraction of the in-band power that actually initiates a photocurrent in the detector material.

Since the wavelength dependence of the PSF within a filter band is small, we simplified Eq. (1) to

$P_{\mathrm{src}}=A f_{\mathrm{PSF}_{\mathrm{f}, \mathrm{a}}} T_{\mathrm{r}} \int\left[T_{\mathrm{f}}(\lambda) R_{\mathrm{p}}(\lambda) F_{\lambda}(\lambda)\right] \mathrm{d} \lambda$.

by replacing the integral over the aperture and the PSF by two factors: (i) The area of the telescope aperture, $A$, and (ii) the PSF-correction factor, $f_{\mathrm{PSF}_{\mathrm{f}, \mathrm{a}}}$, dependent on filter, f, and aperture, a. This second factor describes - for a given combination of filter and aperture - the fraction of the PSF that actually enters the detector aperture.

For the initial calculations we used an analytical telescope model, taking into account the primary and secondary mirrors. Subsequently we replaced this by a

${ }^{5}$ The integral of the PSF over the focal plane is normalized to 1 . 
more accurate model (Okumura 2000) based on numerical Fourier transformation, that also includes effects of the support structure of the secondary mirror. The PSF factors were calculated as the ratio of the in-band power resulting from Eq. (1), integrated over the detector aperture and the total in-band power in the PSF, i.e. Eq. (1) integrated over an infinite aperture. A $\nu F_{\nu}=$ const. spectrum was adopted as source SED.

Thus far, our intent has been to determine the in-band fluxes of known standards to characterise the detectors and to calibrate the FCS. Once the FCS is calibrated, however, we can use the instrument to determine the inband powers of other celestial sources. To convert these back to flux densities, we must invert Eq. (2). For regular target observations the source SED is generally unknown so we need a common reference spectrum with a defined colour. We adopt a spectrum of the form $\nu F_{\nu}=$ const. as Beichman et al. (1988) did for IRAS. This enables us to replace Eq. (2) by the much simpler form

$P_{\mathrm{src}}=F_{\nu} C 1_{\mathrm{f}} f_{\mathrm{PSF}_{\mathrm{f}, \mathrm{a}}}$,

where $C 1_{\mathrm{f}}$ are tabulated factors for the "reference wavelength" (see Table 2) of each filter. The photometric results, $F_{\nu}$, provided by the off-line processing software (OLP) or the interactive analysis (PIA) to the observer are expressed in terms of flux densities at the proper reference wavelength of the filter used. The choice of the reference wavelength is arbitrary, as long as the reference spectrum is defined. For sources with colours deviating from the reference spectrum, a colour correction must be applied, that can be derived from Eqs. (2) and (3). Tabulated colour correction factors were derived for a few typical spectra like blackbodies or power-laws. Table 2 shows the reference wavelengths that are used to calculate ISOPHOT flux densities.

\subsection{Observations}

The ISO observations to calibrate the internal sources were scheduled in blocks for each filter band. Each block comprised measurements of a celestial source, celestial background, heated FCS and non-heated FCS. The measurements were obtained in different sequences, but in general the low-flux measurements were executed before the high-flux measurements to reduce the time constants of transients. These are shorter for transitions from low to high flux. The observations for the long-wavelength detectors P3, C100 and C200 also included direct calibrations of the redundant FCS 2. For the other filter bands, FCS 2 was calibrated relatively to FCS 1 .

To check the relative effective transmission of filter bands, measurement blocks of the same detector were further grouped together, so that responsivity drifts would be minimal during that time. We verified this by repeating the first measurement of the sequence at the end. The results lead to corrections to the filter transmissions, that are discussed in Sect. 6.2.
Table 2. The reference wavelengths, conversion factors, standard apertures and the calibrated flux-range for the 25 ISOPHOT filter bands. The calibrated flux-range is where FCS 1 was directly calibrated against external standards. For revolutions prior to 94 sometimes smaller ranges apply. Extrapolations are possible but may have larger absolute errors. For the C-subsection values are given for the central pixel of C100 and pixel 1 of C200.

\begin{tabular}{lccccc}
\hline Filter & $\begin{array}{c}\lambda_{\text {ref. }} \\
{[\mu \mathrm{m}]}\end{array}$ & $\begin{array}{c}\text { C1 } \\
{\left[10^{11} \mathrm{~m}^{2} \mathrm{~Hz}\right]}\end{array}$ & $\begin{array}{c}\text { Apert. } \\
{\left[{ }^{\prime \prime}\right]}\end{array}$ & $\begin{array}{c}\text { lo lim } \\
{[\mathrm{Jy}]}\end{array}$ & $\begin{array}{c}\text { hi lim } \\
{[\mathrm{Jy}]}\end{array}$ \\
\hline P_3P29 & 3.3 & 1.473 & 23 & 0.410 & 214.7 \\
P_3P6 & 3.6 & 7.535 & $"$ & 0.156 & 788.7 \\
P_4P85 & 4.8 & 7.612 & $"$ & 0.092 & 1196.9 \\
P_7P3 & 7.3 & 12.619 & $"$ & 0.058 & 1922.8 \\
P_7P7 & 7.7 & 2.476 & $"$ & 0.128 & 4331.8 \\
P_10 & 10.0 & 4.495 & 52 & 0.041 & 787.4 \\
P_11P3 & 11.3 & 1.314 & $"$ & 0.543 & 371.0 \\
P_11P5 & 12 & 16.093 & $"$ & 0.097 & 1701.6 \\
P_12P8 & 12.8 & 5.216 & $"$ & 0.845 & 389.3 \\
P_16P0 & 15 & 3.166 & $"$ & 0.078 & 706.3 \\
\hline P_20 & 20 & 5.198 & 79 & 0.696 & 1045.4 \\
P_25 & 25 & 4.277 & $"$ & 1.250 & 1875.9 \\
\hline P_60 & 60 & 0.591 & 180 & 0.631 & 307.7 \\
P_100 & 100 & 0.872 & $"$ & 2.607 & 719.9 \\
\hline C_50 & 65 & 0.481 & 43.5 & 0.741 & 276.5 \\
C_60 & 60 & 0.659 & $"$ & 1.036 & 95.8 \\
C_70 & 80 & 0.697 & $"$ & 0.576 & 167.6 \\
C_90 & 90 & 1.510 & $"$ & 0.245 & 47.8 \\
C_100 & 100 & 0.945 & $"$ & 0.094 & 49.5 \\
C_105 & 105 & 0.611 & $"$ & 0.110 & 172.7 \\
\hline C_120 & 120 & 0.355 & 89.4 & 1.257 & 560.9 \\
C_135 & 150 & 0.711 & $"$ & 1.445 & 363.1 \\
C_160 & 170 & 1.010 & $"$ & 0.837 & 152.6 \\
C_180 & 180 & 0.537 & $"$ & 2.444 & 336.7 \\
C_200 & 200 & 0.233 & $"$ & 1.612 & 532.2 \\
\hline
\end{tabular}

All detector pixels were calibrated individually. Therefore, measurements of the standard point sources were positioned at the centre of every pixel. To minimize the measurement time for the camera arrays, the spacecraft performed a raster mode pointing sequence with a step size equal to the distance between pixel centres $\left(46^{\prime \prime}\right.$ for $\mathrm{C} 100,92^{\prime \prime}$ for C200). Larger raster dimensions $(3 \times 5$ for $\mathrm{C} 100$ and $2 \times 7$ for $\mathrm{C} 200)$ were chosen to obtain the background zero level also. Where no raster measurement was required, background measurements were performed in staring mode on positions within a $5^{\prime}$ radius of the source. For aperture-photometry of faint sources, a onedimensional raster of 3 points with the source at the centre position was scanned back and forth (nodding-mode), to enable elimination of the baseline drift (see Fig. 4).

The use of a raster observation on a fixed sky position was also necessary because ISO could neither observe positions with a relative offset from a tracked Solar System Object (SSO), nor perform raster observations while tracking. Therefore, all raster observations on SSOs had to be scheduled at fixed times with the telescope 
pointing adjusted to encounter the moving object. We made sure that the proper motion of the SSO due to the relative motion w.r.t. ISO was always below $60^{\prime \prime} / \mathrm{hr}$ during observations. The contribution from ISO's motion relative to the centre of the Earth was only of the order of $\pm 3^{\prime \prime} / \mathrm{hr}$, as science observations were made during the "slow" part of the orbit. During the maximum duration of a raster measurement on an SSO with C100 (688 s), asteroids would travel only $\approx 12^{\prime \prime}$. Taking into account ISO's absolute pointing accuracy for fixed targets of about $\pm 2^{\prime \prime}$, the worst case pointing error amounts to $6^{\prime \prime}+2^{\prime \prime}=8^{\prime \prime}$, which is still much smaller than the large detector apertures or pixel sizes used at long wavelengths.

\section{Photometric corrections}

All corrections so far applied to the signal did not require quantitative knowledge of the IR flux illuminating the detector. However, in this section we will describe corrections that became necessary after a detailed photometric analysis of observations of known celestial calibration standards of various brightnesses.

\subsection{Signal linearisation}

We defined earlier the responsivity, $R$, as a property of the detector that is expected to vary in time due to cosmic radiation and flux history, but not to depend on flux level. However, the actual behaviour of ISOPHOT detectors was found to be different.

In its simplest form we calculate the responsivity from the signals measured on-source $\left(S_{\mathrm{src}}+S_{\mathrm{bck}}\right)$ and off-source $\left(S_{\mathrm{bck}}\right)$ and the PSF-corrected in-band power, $P_{\mathrm{src}}$, according to

$R=\frac{S_{\mathrm{src}} C_{\mathrm{int}}}{P_{\mathrm{src}}}$,

where $C_{\text {int }}$ is the capacity of the integrating amplifier. Plotting responsivities from measurements of various calibration standards versus in-band power, however, shows strong correlations (see Fig. 9). Moreover these correlations are different even for filters of the same detector subsystem.

If the measured responsivity depends in some way on the IR flux that falls onto the detector, $R$ can also be expressed as a function of the photocurrent or the detector signal. We then rewrite Eq. (4) as

$R\left(S_{\mathrm{src}}+S_{\mathrm{bck}}\right)=\frac{\left(S_{\mathrm{src}}+S_{\mathrm{bck}}\right) C_{\mathrm{int}}}{P_{\mathrm{src}}+P_{\mathrm{bck}}}$,

describing the responsivity of the detector when it is pointed at the position of the source, and further as

$R\left(S_{\mathrm{src}}+S_{\mathrm{bck}}\right)=\frac{\left(S_{\mathrm{src}}+S_{\mathrm{bck}}\right) C_{\mathrm{int}}}{P_{\mathrm{src}}+\frac{S_{\mathrm{bck}} C_{\mathrm{int}}}{R\left(S_{\mathrm{bck}}\right)}}$.

The index bck indicates the signal and in-band power at the background position. In our case, $R\left(S_{\mathrm{bck}}\right)$ and
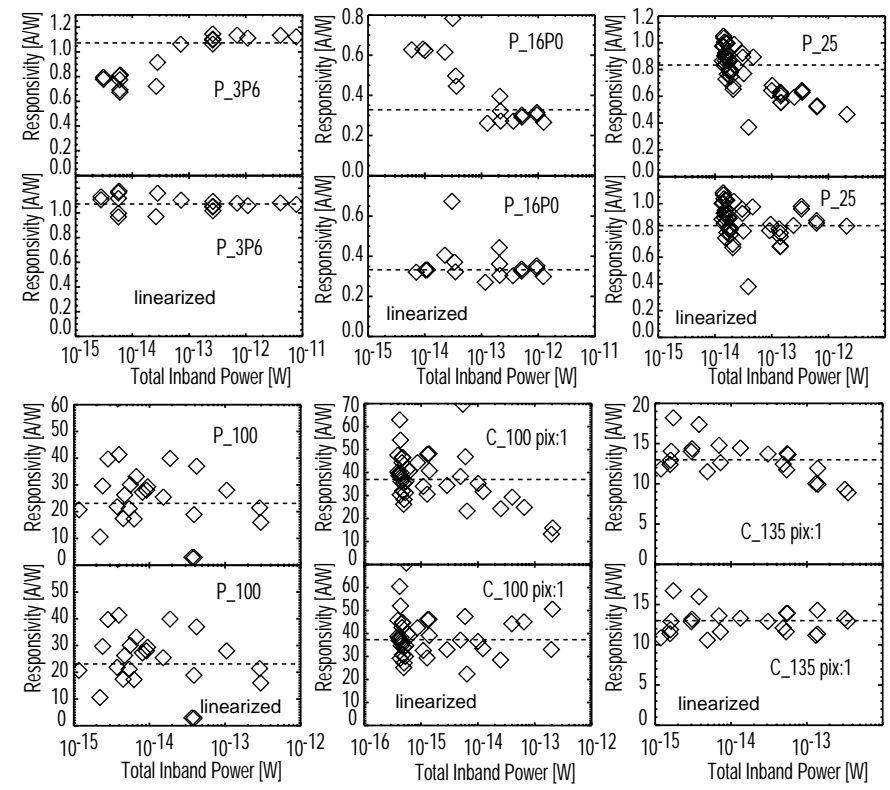

Fig. 9. Naive plots of responsivities against total in-band power, assuming linearity (Eq. (4)) for representative filter bands of all detectors. The total in-band power includes the contributions from both source and background. Upper and lower diagrams show the responsivities before and after linearisation. The upper diagrams show a clear dependence on in-band power except for P3. The remaining scatter in the linearised data is due to the variation of the responsivity with time.

$R\left(S_{\mathrm{src}}+S_{\mathrm{bck}}\right)$ are not necessarily equal and the ratio of the signals measured on- and off-source is different from the ratio of the respective in-band powers. To determine $R(S)$ requires knowledge about $R\left(S_{\mathrm{bck}}\right)$ because of Eq. (6). Considerable errors are introduced in comparisons of very different signals if $R$ is assumed to be independent of signal. This applies in particular to multifilter AOTs, multi-aperture AOTs on extended sources, maps with large dynamic ranges, and all FCS calibrations where the signals from FCS and sky are very different. For measurements on the sky, the biggest systematic errors are expected when the individual contributions of background and source alone are about equal.

All further data reduction steps assume a linear system, so we decided to linearise the detector signals, $S$, according to $S^{\prime}=\mathcal{T}(S)$, where $\mathcal{T}$ is a continuously rising transfer function. The condition for $\mathcal{T}(S)$ is such that the results of $S^{\prime}$ inserted into Eq. (4) should no longer correlate with the detector signal, but should be distributed around a constant responsivity with minimum dispersion. The absolute value of this constant is arbitrary because it cancels out in the flux calibration. We normalized it to the median of the nonlinearised responsivities calculated according to Eq. (4). It should be noted that application of the signal linearisation requires the prior subtraction of the dark signal.

Although the FCS calibration data comprise a large number of measurements, the number of independent responsivities distributed over the entire flux range per filter 

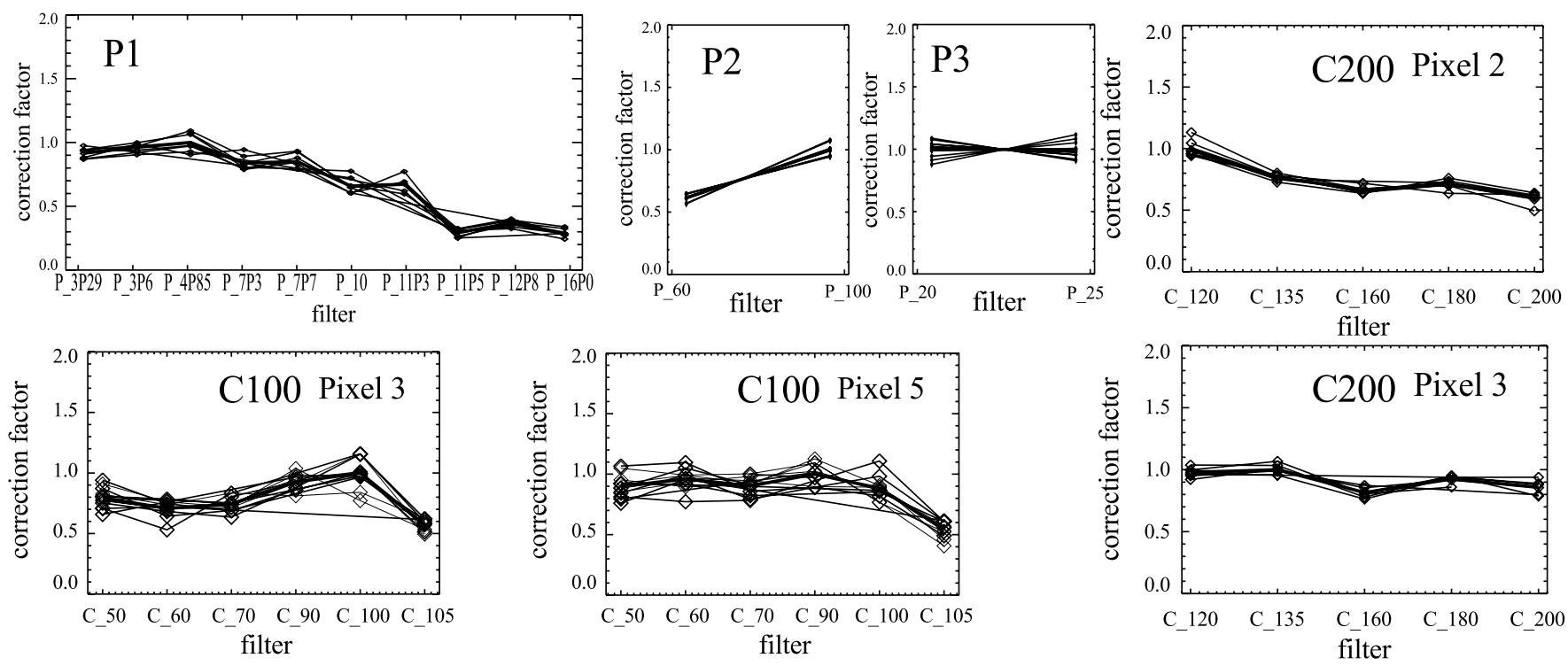

Fig. 10. Derivation of filter-to-filter corrections. We display the data for some representative detectors. Each diagram shows the normalized responsivities for the indicated detector, derived from various filter sequences. Each sequence was measured on a calibration standard within a time interval, sufficiently short to avoid long term drifts. Each sequence is connected by a line. The standard deviation of the scatter of the relative filter measurements is 2 to $9 \%$ for the P-detectors and 3 to $8 \%$ for the C-arrays.

band ranges only between 14 and 28 . We developed a manual fitting method to determine the linearisation tables, by displaying the results of Eq. (6) and interactively modifying the linearisation function, $\mathcal{T}(S)$. This approach led to a better exploration of parameter space, a smoother transition at the boundaries, and a more tolerant treatment of outliers without the need to develop sophisticated fitting algorithms. We increased the objectivity of the method by keeping the shape of $\mathcal{T}$ simple, generally close to a polynomial of second or third order. We also minimised differences between pixels of the array detectors by fitting all pixels of one detector in parallel on the same screen. In spite of the large scatter of responsivities, simple functions $\mathcal{T}$ were found that significantly increase the consistency of data measured at different flux levels.

Signal linearisation is performed by interpolation in a lookup table spanning the full range of possible signals. This table is calibrated only in the range covered by calibration standards but, in practice, signals occur outside this range that are higher, lower, or even negative in the case of noisy measurements close to the dark current. Reasonable entries for these cases had to be defined. For high signals beyond the point where data are available, we maintained a constant responsivity. Small signals are constrained so that an already dark-subtracted zero signal is not changed by signal linearisation. Therefore, we continue $\mathcal{T}(S)$ between the smallest point in the lookup table that is still determined by valid data, and zero, by a straight line. This assumes that the responsivity does not vary in that range. c) The most difficult cases are negative signals, because negative fluxes are simply undefined and appear only as a result of noise. The number of such cases actually encountered is low due to the reduced noise after signal averaging over periods of constant flux (SCP level). We decided to continue the transfer function such that $\mathcal{T}(S)=-\mathcal{T}(-S)$, to cover all situations that occur.

\subsection{Filter-to-filter calibration}

Calculating the detector responsivity from standard source observations according to

$R_{\mathrm{p}, \mathrm{f}}^{\prime}=\frac{\left(S_{\mathrm{src}_{\mathrm{p}, \mathrm{f}}}-S_{\mathrm{bck}, \mathrm{f}}\right) C_{\mathrm{int}}}{P_{\mathrm{f}}}$,

after application of all signal corrections described so far, still yields different results for the same detector pixel, p, measured in different filters, f, during the same batch of observations. The ratios of responsivities between filters are reproducible for different calibration sources and cannot be explained by responsivity variations with time.

The worst case is detector $\mathrm{P} 1$, where $R$ varies by a factor of 4 between filters P_3.29 and P_16 (see Fig. 10). Currently, the wavelength-dependent flux losses of this detector remain unexplained. We speculate, however, that the sapphire substrate that mechanically supports the detector crystal within the kidney-shaped aluminium cavity might play a role. Sapphire is transparent in the NIR and starts to absorb beyond about $6 \mu \mathrm{m}$ onwards, in the middle of P1's wavelength range (Tropf \& Thomas 1998). The absorption peak is at $\approx 17.5 \mu \mathrm{m}$. Some fundamental lattice vibration modes occur in the reststrahlen region above $13 \mu \mathrm{m}$. Top and bottom of both detector and substrate are gold-coated. The remaining effective area of the detector, where radiation from within the cavity can enter, is only twice that of the substrate. Given the high refractive index of $\mathrm{Si}$, around 3.4, and the consequently 
higher reflectivity compared to $\mathrm{Al}_{2} \mathrm{O}_{3}$ (refractive index of only 1.7), we estimate that about the same number of photons enters both detector and substrate. The cavity was designed to counteract the high refractive index of the detector material by maximizing the number of reflections within it. Assuming that the sapphire substrate turns "dark" at longer wavelengths, it is conceivable that, with every reflection, a substantial fraction of the radiation enters the substrate and is attenuated. The effective response of the detector system would thus deteriorate. Another IR absorber present within the cavity is glue (Stycast). Unfortunately the question of P1's quantum efficiency remains largely academic, so that the effort of a much more detailed analysis is not justified.

The other detectors show less dramatic variations by factors up to 2. Moreover, the ratios found between different filters are not the same for different pixels of the same $\mathrm{C}$-detector array. We attribute this to a projection of spatial nonuniformities of the filter surface onto the detector array, because the ISOPHOT filters are not located at the pupil of the optical path, but close to the detectors. The overall variation between filters of all detectors, except P1, is most likely due to optical misalignments and diffraction effects that are neglected in our ideal instrument model.

We applied a correction to the responsivity by introducing a matrix, $\chi_{\mathrm{p}, \mathrm{f}}$. It works equally for P- and $\mathrm{C}$-detectors. To obtain a wavelength independent detector responsivity, we split $R^{\prime}$ of Eq. (7) into filter-independent and filter-dependent parts, so that $R_{\mathrm{p}} \chi_{\mathrm{p}, \mathrm{f}}=R_{\mathrm{p}, \mathrm{f}}^{\prime}$. The matrix, $\chi_{\mathrm{p}, \mathrm{f}}$, was established from multi-filter measurements on calibration standards, executed so that the change of responsivity with time could be neglected for a filter sequence. After eliminating poor quality measurements, the responsivities measured for a detector pixel, $\mathrm{p}$, in a filter sequence were renormalized. The factors per sequence were chosen to achieve the best match among all sequences for a given pixel. After calculating the average responsivities, $R_{\mathrm{p}, \mathrm{f}}$, per filter, $\mathrm{f}$, we normalized to the maximum responsivity found for that pixel using $\chi_{\mathrm{p}, \mathrm{f}}=R_{\mathrm{p}, \mathrm{f}} / \max \left(R_{\mathrm{p}, \mathrm{f}}\right)$, thus assuming that the matrix describes flux losses w.r.t. the ideal model. The normalized data and the resulting filter-to-filter corrections, $\chi_{\mathrm{p}, \mathrm{f}}$, for some detector pixels and filter bands, are shown in Fig. 10. The standard deviation of the scatter of the relative filter measurements is 3 to $8 \%$ for the C-arrays and 2 to $9 \%$ for the P-detectors.

\section{Derivation of FCS power curves}

The FCSs were calibrated against celestial standards in all 25 filter bands. These measurements yield the relationship between in-band power, $P$, and FCS heating power, $\mathrm{h}$, for all combinations of filter band, f, detector pixel, p, and FCS. Signals are measured differentially. The source signal is obtained from the difference between on-source, $S_{\text {src }}$, and off-source, $S_{\mathrm{bck}}$, signals while the FCS signal is calculated as the difference between the signals measured with heated $\operatorname{FCS}\left(S_{\mathrm{FCS}}\right)$ and cold $\operatorname{FCS}\left(S_{\text {str }}\right)$. Since the

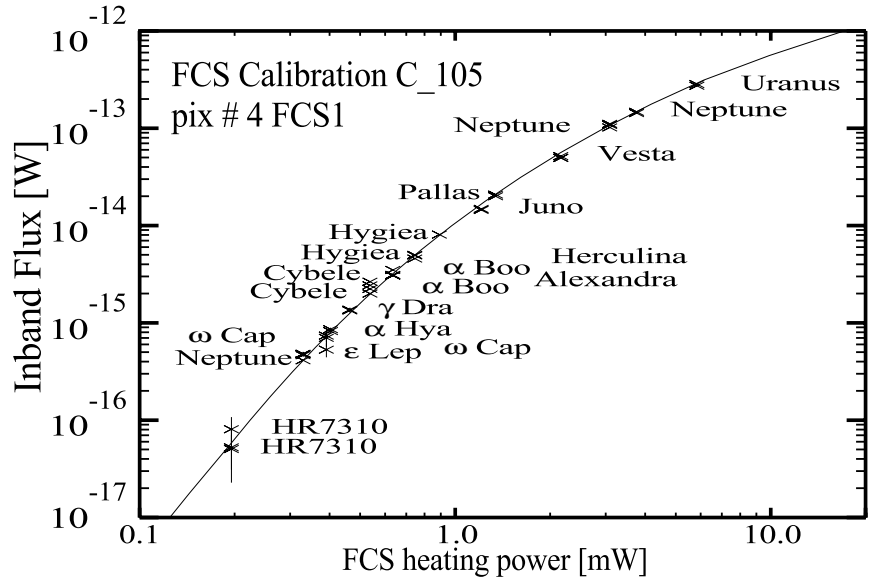

Fig. 11. Constructing a power curve from calibration data. The names indicate the sources that have been used to calibrate the respective data points.

measurements of FCS and celestial standard were performed in the same filter, the responsivity does not need to be explicitly calculated. We used

$P_{\mathrm{FCS}}^{\prime}(h)=\frac{\left(S_{\mathrm{FCS}}(h)-S_{\mathrm{str}}\right) P_{\mathrm{src}}}{S_{\mathrm{src}}-S_{\mathrm{bck}}}$,

where $P_{\mathrm{FCS}}^{\prime}(h)$ is the in-band power incident on the detector from the FCS and $P_{\mathrm{src}}$ is defined in Eq. (2). Figure 11 shows an example of the power curve resulting from observations of different calibrators for pixel 4 in the C_105 filter band.

\subsection{Illumination matrices and FCS apertures}

FCS power curves for different pixels of the same C-array differ by constant factors. These factors result from both the nonuniform illumination of the detector arrays by the FCS and the putative spatial inhomogeneities in the filter. For each filter, these factors constitute the illumination matrix, $\Gamma_{\mathrm{p}, \mathrm{f}}$, as shown in Fig. 12. Thus the FCS curve for each filter is split into a pixel-independent part, $P_{\mathrm{FCS}_{\mathrm{f}}}(h)$, and a pixel-dependent factor, $\Gamma$, so that $P_{\mathrm{FCS}_{\mathrm{p}, \mathrm{f}}}^{\prime}(h)=\Gamma_{\mathrm{p}, \mathrm{f}} P_{\mathrm{FCS}_{\mathrm{f}}}(h)$. The FCS curve is normalized such that $\Gamma_{\mathrm{p}, \mathrm{f}}$, averaged over all pixels becomes 1 , for a given filter. The resulting FCS power curves for FCS 1 are shown in Figs. 13 and 14. Between revolution 93 and 94 the TRS 2 of FCS 1 , which was routinely used with the long-wavelength detectors, abruptly increased its brightness by about a factor of 2 . This change necessitated a recalibration of the corresponding filter bands and resulted in a second set of FCS calibration tables for P3, C100 and C200. Figure 14 shows the FCS power curves that apply to the period before revolution 94 . The non-uniformity of the FCS illumination also affects the FCS signal measured in different apertures of the P-detectors. For a fixed heating power the FCS signal is not proportional to the area of the aperture. Strong deviations exist for apertures much larger or smaller than the standard apertures. The treatment of these cases is still poorly understood and is 


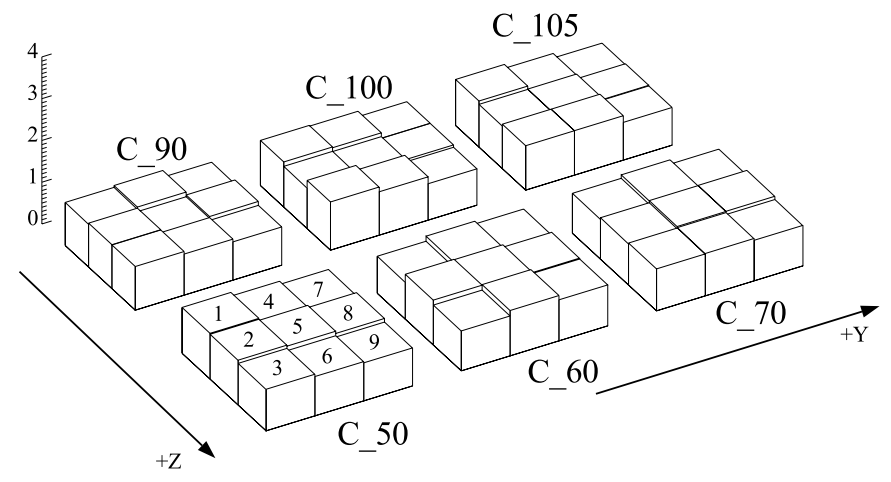

C_200

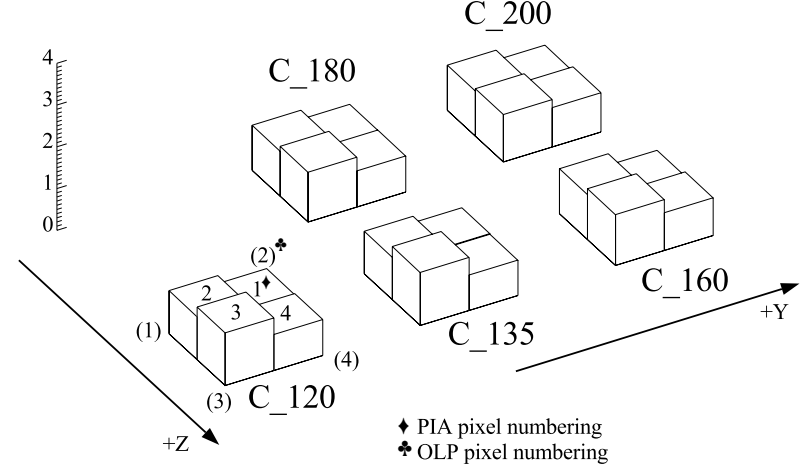

Fig. 12. Relative illumination of the array detectors C100 and C200 (illumination matrices) by FCS 1 (valid after rev. 93). Pixel numbers and ISO coordinate axes are given.

beyond the scope of this paper. For use with standard and very similar apertures, the in-band powers of the PHT-P power curves were divided by the area of the standard aperture of the measurement, and are expressed in units of $\mathrm{W} / \mathrm{mm}^{2}$.

\subsection{Extrapolation}

During the mission, FCS heating powers were used that fell beyond the calibrated range of heating powers. We used the grey body model described in Sect. 3 to extrapolate the power curves. Including the spectral transmission of the beam splitter of the FCS and replacing the celestial source SED by the grey body spectrum, the inband powers are calculated in accordance with Eq. (2). The free parameters $\alpha$ and $\beta$ and the effective solid angles of the detector subsystems are obtained from least-squares fits to the FCS curves (Schulz 1993). Comparison of the model to the in-orbit data shows good consistency, especially at longer wavelengths. At shorter wavelengths the consistency is poorer. Extending the measured FCS power curves by the models provided the values outside the range of measured heating powers. This range is flagged in the associated calibration tables.

\subsection{FCS straylight}

Ground calibrations already showed an increased signal compared to the dark signal when measuring the signal from a "cold" FCS. Considering the internal ISOPHOT geometry, this additional signal is most likely picked up from radiation within the field of view of the telescope (Schulz 1993). The in-orbit observations, where the cold FCS signal was found to be correlated with the background signal, confirm the presence of this kind of straylight for C200 (Cornwall 1997). C100 and P3 show no detectable straylight component. $\mathrm{P} 2$ shows a constant value of $0.02-0.04 \mathrm{~V} / \mathrm{s}$ for a signal $>0.2 \mathrm{~V} / \mathrm{s}$ (on source) and a steep fall-off for lower signals. Straylight in P1 is always below $0.07 \mathrm{~V} / \mathrm{s}$ but shows a nonlinear dependence on signal. The nonlinear dependences for both detectors $\mathrm{P} 1$ and $\mathrm{P} 2$ are quite uncertain but appear to be due to the varying dark signal and systematic errors in the transient fit at low signals, rather than to straylight, which is expected to be proportional to the intensity of the incident flux components. We conclude, therefore, that FCS straylight should be taken into account for C200, but can be neglected for the other cases. We determined the straylight at the FCS position measured with C200 to be 5\% of the background level.

\section{Flux calibration}

We can now detail the procedure to calibrate ISOPHOT observations. First, the detector responsivity is determined from the FCS measurement using

$R_{\mathrm{p}}=\frac{\left(S_{\mathrm{FCS}_{\mathrm{p}, \mathrm{f}}}-S_{\mathrm{str}_{\mathrm{p}, \mathrm{f}}}\right) C_{\mathrm{int}}}{P_{\mathrm{FCS}_{\mathrm{f}}}(h) \Gamma_{\mathrm{p}, \mathrm{f}} \chi_{\mathrm{p}, \mathrm{f}}}$.

$P_{\mathrm{FCS}_{\mathrm{f}}}(h), \Gamma_{\mathrm{p}, \mathrm{f}}$ and $\chi_{\mathrm{p}, \mathrm{f}}$ are taken from the calibration tables. The FCS straylight signal $S_{\mathrm{str}_{\mathrm{p}, \mathrm{f}}}$ was actually measured only in absolute photometry AOTs and in dedicated FCS calibration measurements. In all other cases it is replaced by the time-dependent dark signal (see Sects. 4.7 and 7.3). C200 should be corrected for the 5\% straylight contribution of the background. Second, the in-band power, $P_{\mathrm{f}}$, for all filters of the detector subsystem is calculated as

$P_{\mathrm{p}, \mathrm{f}}=\frac{S_{\mathrm{src}_{\mathrm{p}, \mathrm{f}}} C_{\mathrm{int}}}{R_{\mathrm{p}} \chi_{\mathrm{p}, \mathrm{f}}}$.

The transformation to a flux density, $F_{\nu}$, at the reference wavelength of the filter (e.g. $12 \mu \mathrm{m}$ for the P_11.5 filter) is done by changing Eq. (3) to

$F_{\nu_{\mathrm{p}, \mathrm{f}}}=\frac{P_{\mathrm{p}, \mathrm{f}}}{C 1_{\mathrm{f}} f_{\mathrm{PSF}_{\mathrm{f}, \mathrm{a}}}}$.

The flux density of the point source is obtained by subtracting the flux density derived from a measurement on the background, following the same calibration steps as for the source observation. The resulting flux density is calculated at the reference wavelength of the filter for an assumed $\nu F_{\nu}=$ const. spectrum and still has to be colourcorrected in accord with the actual source spectrum. It should be noted that, when the point source is not placed at the centre of the detector pixel or aperture, a different 

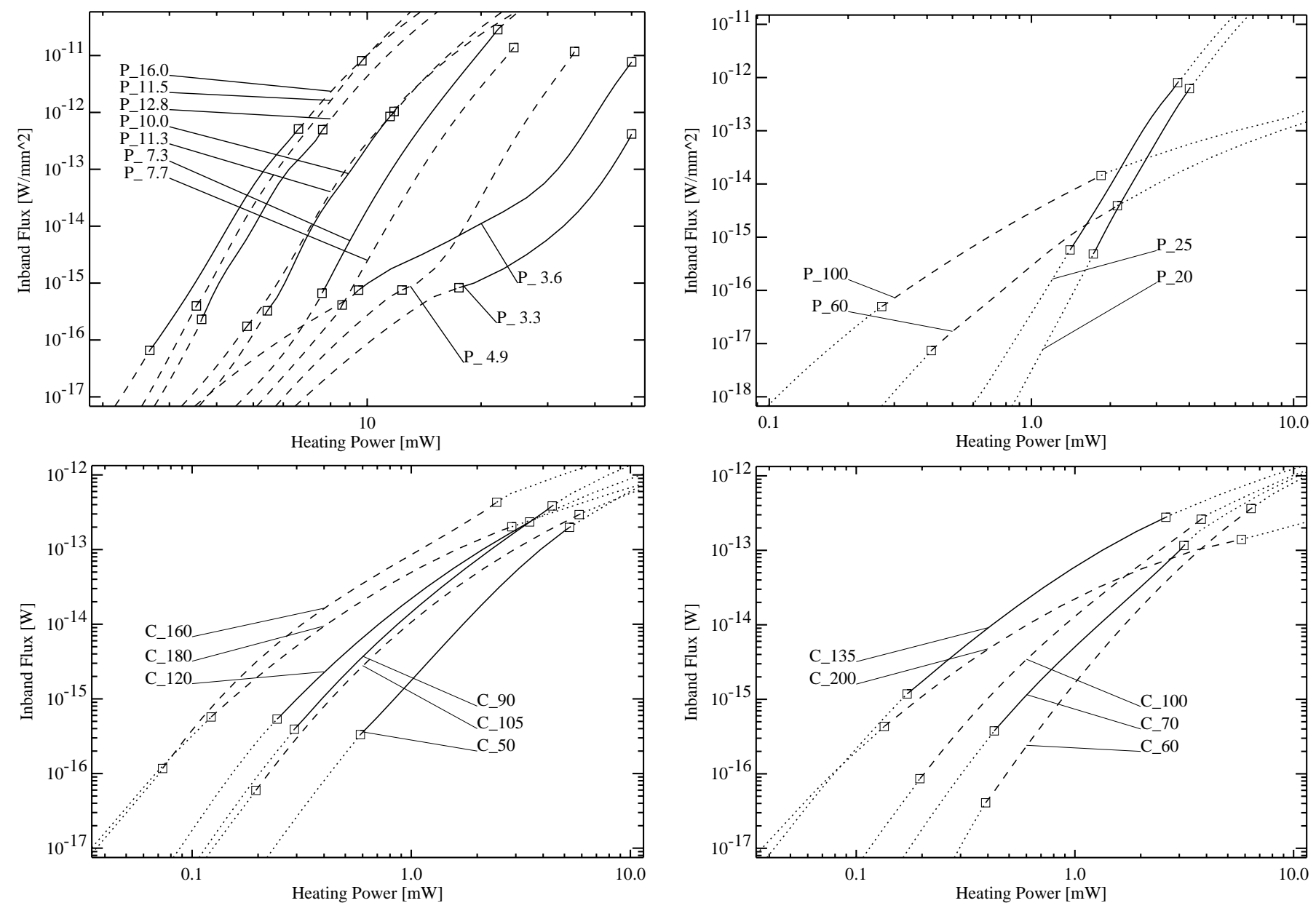

Fig. 13. FCS power curves of all filter bands. For P3, C100 and C200 we show the versions valid after the change of FCS $1 /$ TRS 2 in rev. 94. The interpolated parts are indicated by solid or dashed lines with small squares at the boundaries, while the model extrapolations are indicated by dotted lines.
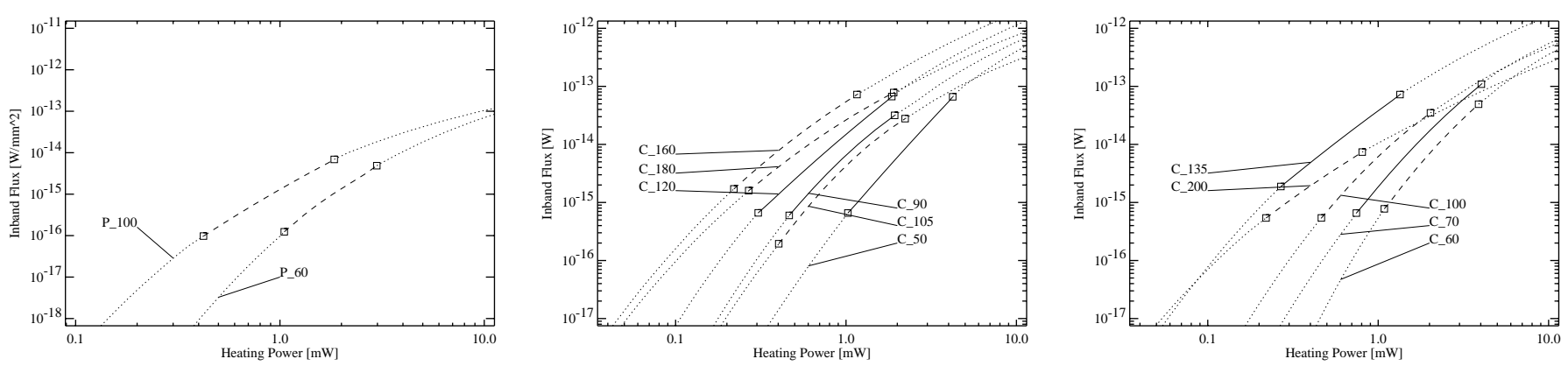

Fig. 14. FCS power curves of all filter bands of the long wavelength detectors before the change in FCS $1 /$ TRS 2 .

PSF correction factor must be applied. In particular, in observing modes with C-detectors, the sum of the fluxes found in all pixels can be calculated and a PSF correction factor, $f_{\mathrm{PSF}_{\mathrm{f}, \mathrm{a}}}$, determined for the entire array, is applied.

\subsection{Accuracy}

We assess the overall consistency of the point source calibration in standard apertures by comparing the measured FCS in-band fluxes for all available heating powers with the FCS power curves. The scatter is representative of the error budget, except for any systematic biases in the SEDs of the calibration sources. Each measurement passed through the standard signal processing chain and thus contains the same uncertainties as the science observations. The additional automatic transient fit we applied during the calibration data processing is equivalent to improvements the general observer can achieve through manual processing by Interactive Analysis. We plot the ratio of the in-band power taken from the calibration tables for the FCS (FCS power curves times FCS illumination tables) and the measured FCS in-band powers. To indicate 
Table 3. Comparison of derived FCS power curves with measured calibration data. The deviations are given in percent. $n$ is the number of measurements used, max is the maximum deviation found and $\sigma$ is the rms deviation. The last two columns $\sigma_{\text {lo }}$ and $\sigma_{\text {hi }}$ give the rms deviations in the low and high flux regions separated by the vertical dashed lines in Figs. 15 and 16.

\begin{tabular}{lccccc}
\hline Filter & $n$ & $\begin{array}{c}\max \\
{[\%]}\end{array}$ & $\begin{array}{c}\sigma \\
{[\%]}\end{array}$ & $\begin{array}{c}\sigma_{\text {lo }} \\
{[\%]}\end{array}$ & $\begin{array}{c}\sigma_{\text {hi }} \\
{[\%]}\end{array}$ \\
\hline P_3P29 & 5 & 6 & 4 & 4 & - \\
P_3P6 & 13 & 17 & 6 & 8 & 1 \\
P_4P85 & 10 & 27 & 10 & - & 3 \\
P_7P3 & 19 & 26 & 8 & 11 & 4 \\
P_7P7 & 17 & 15 & 5 & 5 & 5 \\
P_10 & 11 & 21 & 8 & - & 6 \\
P_11P3 & 6 & 15 & 8 & - & 8 \\
P_11P5 & 26 & 42 & 13 & 18 & 4 \\
P_12P8 & 15 & 19 & 8 & 9 & 7 \\
P_16P0 & 16 & 40 & 15 & 12 & 17 \\
\hline P_20 & 17 & 14 & 7 & 4 & 4 \\
P_25 & 32 & 19 & 10 & 9 & 10 \\
\hline P_60 & 75 & 82 & 20 & 22 & 12 \\
P_100 & 27 & 52 & 25 & 27 & 22 \\
\hline C_50 & 372 & 53 & 15 & 14 & 16 \\
C_60 & 330 & 48 & 13 & 15 & 12 \\
C_70 & 332 & 44 & 15 & 11 & 16 \\
C_90 & 250 & 76 & 18 & 15 & 20 \\
C_100 & 621 & 94 & 18 & 19 & 14 \\
C_105 & 295 & 76 & 11 & 14 & 7 \\
\hline C_120 & 154 & 22 & 9 & 10 & 7 \\
C_135 & 158 & 40 & 9 & 10 & 9 \\
C_160 & 239 & 57 & 16 & 18 & 11 \\
C_180 & 157 & 31 & 8 & 8 & 8 \\
C_200 & 153 & 45 & 8 & 10 & 6 \\
\hline & & & & &
\end{tabular}

the flux regimes, we plot the ratios versus in-band power on the detector. All data from a single subinstrument appear together in one diagram. Figures 15 and 16 show the ratios derived for the bulk of the mission for FCS 1 and the ratios that apply for FCS 1/TRS 2 before revolution 94 . As expected, the consistency improves with flux (i.e. the scatter diminishes), because statistical noise becomes less important and time constants of transients decrease.

To obtain another quantitative representation, we considered the deviations for individual filters and computed the maxima and the rms. We also calculated the rms of the portions left and right of the dotted lines in Figs. 15 and 16, to seek any differences between higher and lower fluxes. Table 3 contains the values applicable for most of the mission while Table 4 shows the values for the time before rev. 94. These figures and tables give an overview of the accuracy expected for an observation of a point source in the standard aperture, including measurements of background, source, FCS and cold FCS. Since the FCS straylight measurement can in most cases be replaced by the dark signal, the validity of this assessment extends to even a larger number of observations. For multi-filter measurements, where an FCS measurement in only a single filter
Table 4. Same as previous table but for the period before rev. 94.

\begin{tabular}{lccccc}
\hline Filter & $n$ & $\begin{array}{c}\max \\
{[\%]}\end{array}$ & $\begin{array}{c}\sigma \\
{[\%]}\end{array}$ & $\begin{array}{c}\sigma_{\mathrm{lo}} \\
{[\%]}\end{array}$ & $\begin{array}{c}\sigma_{\mathrm{hi}} \\
{[\%]}\end{array}$ \\
\hline P_60 & 13 & 54 & 21 & 16 & 22 \\
P_100 & 8 & 10 & 7 & - & 8 \\
\hline C_50 & 106 & 35 & 18 & 15 & 19 \\
C_60 & 92 & 58 & 22 & 25 & 19 \\
C_70 & 108 & 29 & 14 & 13 & 14 \\
C_90 & 109 & 31 & 17 & 20 & 14 \\
C_100 & 94 & 39 & 16 & 18 & 12 \\
C_105 & 92 & 41 & 17 & 16 & 17 \\
\hline C_120 & 49 & 17 & 7 & 7 & 6 \\
C_135 & 41 & 16 & 6 & 6 & 7 \\
C_160 & 58 & 23 & 8 & 8 & 8 \\
C_180 & 35 & 13 & 5 & 4 & 6 \\
C_200 & 27 & 15 & 7 & 7 & - \\
\hline
\end{tabular}

is available to determine $R$ (Eq. (9)), this FCS calibration can be transferred to a sky measurement in another filter of the same subsystem, because the ratios of transmissions between filters have been determined (see Sect. 6.2). However, this transfer also accretes the uncertainty of the factor $\chi_{\mathrm{p}, \mathrm{f}}(\approx 10 \%)$ for any filter different from the one that was actually used during the FCS measurement. For a single staring observation performed in a standard aperture, we find scatters in the range from 3 to $29 \%$, depending on detector, filter band and flux. The inhomogeneous distribution of data points suggests that systematic effects still dominate the error budget. Since the data points are few and the properties of the distribution, which may not be Gaussian, also depend on flux or signal level, the results must be interpreted with care. The average scatter $(10 \%)$ over all filters of detector $\mathrm{P} 1$ appears to be the best metric for the accuracy that can be expected, but it is likely to be better. A similar result applies to detector P2. Only P3 shows a much larger scatter of about $20 \%$ as a consequence of its degraded performance in the radiation environment in space compared to the laboratory (Lemke et al. 1996). Better statistics are available for the 9 pixels of $\mathrm{C} 100$, that perform better than P3 although the detector material (Ge:Ga) is the same. We find an average scatter of $13 \%$. A better background subtraction, due to the raster mapping method we applied for the C-detectors, may also contribute to the reduced scatter. The results for the C200 detector also provide good statistics. The average scatter is about $10 \%$, fairly consistent over all filters.

These numbers serve as a guideline for the astronomer as to what accuracies to expect for a staring or raster observation that underwent a complete data reduction, involving all reduction steps and all necessary supporting measurements, i.e. background, FCS, FCS straylight. To assess the uncertainty of the FCS power curves alone, however, they only represent upper limits, since statistical uncertainties decrease with the number of data points used. 

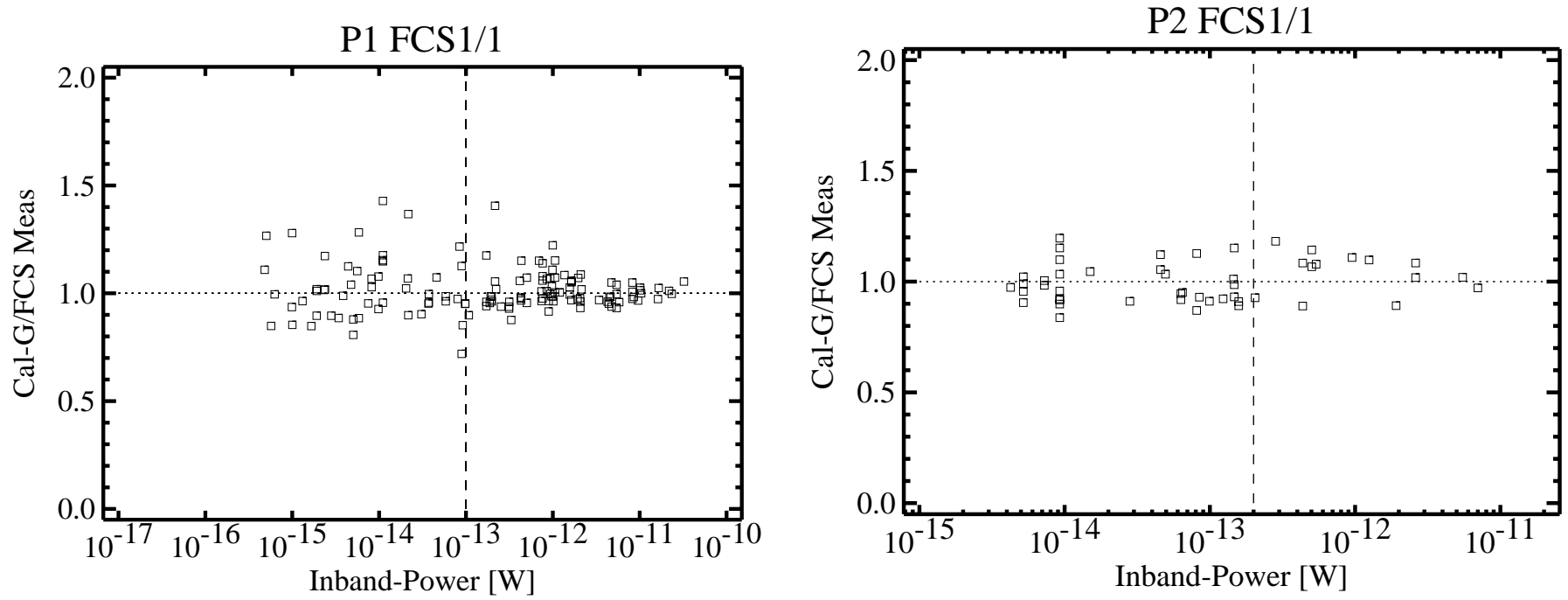

P3 FCS1/2 before rev. 94
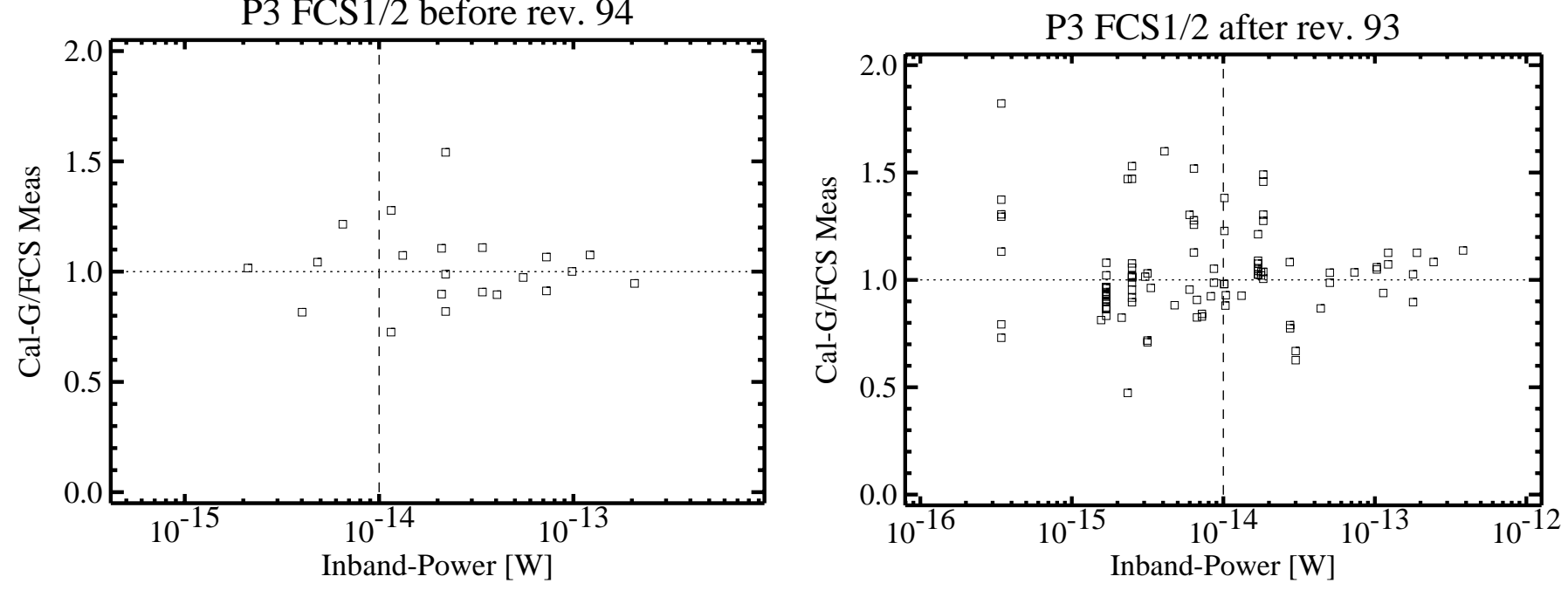

Fig. 15. Calibration accuracy derived from calibration consistency. The diagrams show the ratio of the FCS power curves times the illumination matrices and the data points derived for the P-detectors, plotted against FCS in-band power. The residuals of all filters of a subsystem are combined within one plot. The scatter is a measure of the error budget for single observations. The vertical dashed line separates the low and high flux intervals.

The uncertainties obtained are similar to or larger than the accuracies quoted for the SEDs of the celestial standards. This limits our ability to discriminate problematic standards in comparison to others. A first analysis is given by Schulz (2001).

\subsection{Long-term monitoring}

In order to test the stability and the reproducibility of the system, a set of non-variable celestial sources was monitored at regular intervals throughout the mission. A bright and a faint source were repeatedly measured in the standard filter band of each detector, using the standard apertures in the case of the P-detectors. For the bright source we used the planetary nebula NGC 6543, which was the prime photometric reference for IRAS due to its good visibility and detectability from the MIR to the FIR (Beichman et al. 1988). The faint sources were chosen from the list of stellar calibration standards with good visibility throughout the mission (see Table 5).

The measurements on NGC 6543 were repeated every 5 weeks on average; the fainter sources were measured every 2 weeks. The planetary nebula was measured at the centre of each detector system, after performing a background measurement at a position about $5^{\prime}$ away. The faint sources were observed either in nodding mode for the P-detectors or with a small raster of $3 \times 3$ or $4 \times 2$ for C100 and C200 respectively, to minimize the uncertainties due to background subtraction. We find the reproducibility of measurements on bright sources to be around 2$3 \%$, except for P1 and P3 for which this is $6-7 \%$. The fainter sources show larger scatter because uncertainties of source, background, FCS and FCS straylight contribute equally to the error, compared to the uncertainties only of source and FCS that contribute at higher fluxes. Photon noise and glitch noise also contribute relatively more at 

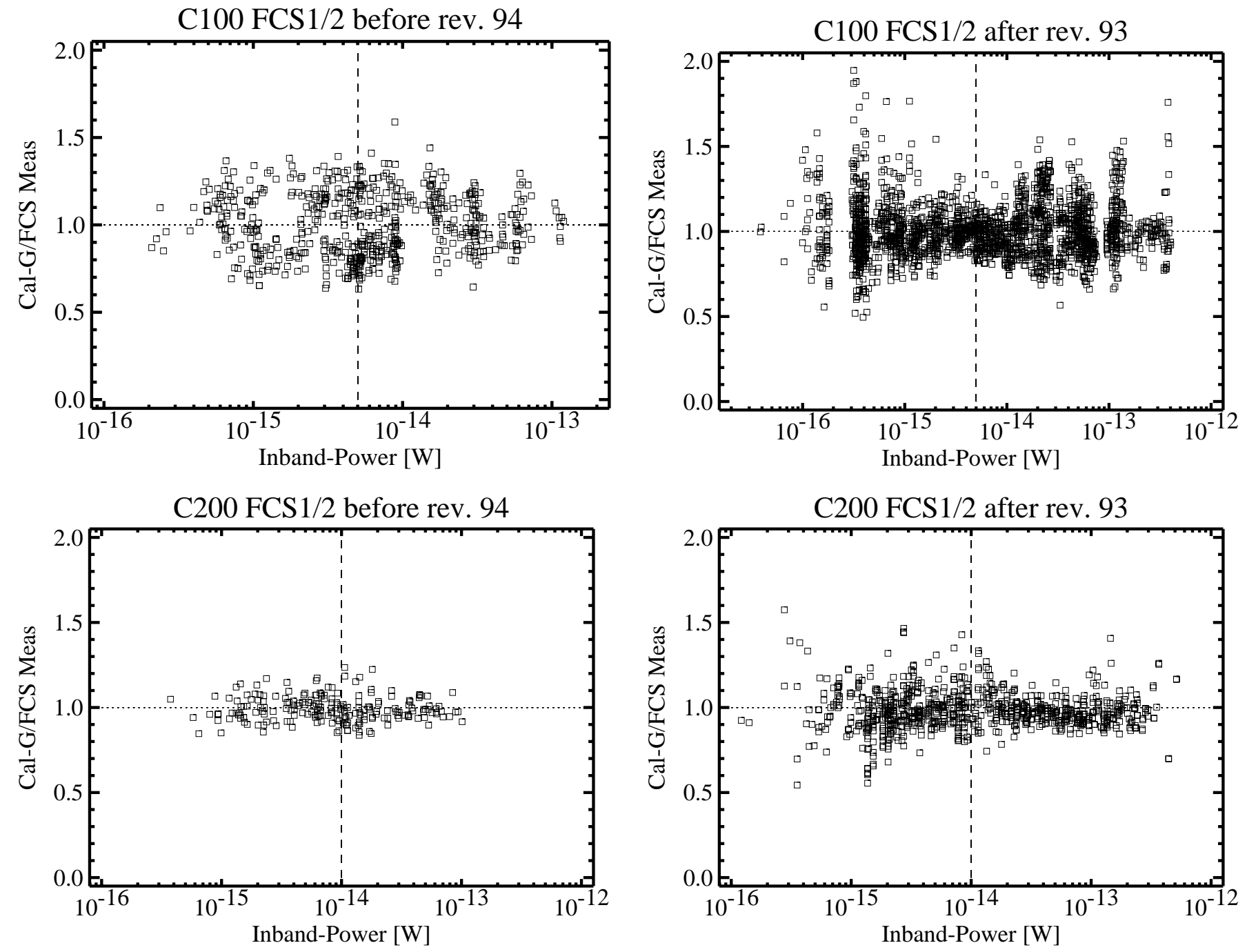

Fig. 16. Calibration accuracy derived from calibration consistency. The diagrams show the ratio of the FCS power curves and the data points derived for the C-detectors, plotted against FCS in-band power. The residuals of all filters of a subsystem are combined within one plot. The scatter is a measure of the error budget for single observations. The vertical dashed line indicates the separation between low and high flux intervals.

low fluxes. The reproducibility below 1 Jy ranges from 7 to $12 \%$. A summary is given in Table 5 .

\section{Summary}

We have described the photometric calibration of ISOPHOT using celestial standards. We used point sources either stars, planets or asteroids, to cover the full wavelength range. Standards at all flux levels were used to calibrate nonlinearities.

Because the detector responsivity varied with time, stable internal IR sources were calibrated against celestial standards and measurements of these references were included in each scientific observation. The reproducibility of results derived from the ratios of these signals was verified for high and low flux levels. We corrected for non-ideal effects like nonlinearities, imperfect optics and detector transients, and reduced their influence on the measurements to a minimum.

The photometric calibration of the internal sources initiated the definition of empirical FCS power curves and FCS illumination tables as well as the introduction of matrices of calibration factors, to account for pixel- and filter-dependent attenuations, which are probably caused by misalignments, diffraction and spatial gradients in filter transmissions. A major step in achieving a consistent picture was the correction for the flux dependence of the responsivity. A set of formulae describing the path from raw detector signals to flux densities was derived, which uses the detector responsivity to characterise the state of a detector pixel at the time of the observation.

To quantify the accuracies achievable by absolute photometry, a comparison was made of the derived calibration curves of the internal sources and the measured data. Depending mainly on flux level, the achievable 
Table 5. Overview of results of the monitoring programme of faint and bright flux standards. The sources, filters and apertures used appear in the first three columns; the fourth and fifth show the flux densities calculated from model SEDs and the observed error-weighted mean values. Flux densities are not colour-corrected, i.e. they assume a $\nu F_{\nu}=$ const. spectrum. The last column shows the standard deviation of the distribution of measurements.

\begin{tabular}{llcccc}
\hline source & filter & apert. & $\begin{array}{c}F_{\nu} \\
{[\mathrm{Jy}]}\end{array}$ & $\begin{array}{c}F_{\nu_{\text {meas. }}} \\
{[\mathrm{Jy}]}\end{array}$ & $\begin{array}{c}\sigma \\
{[\%]}\end{array}$ \\
\hline HD 172323 & P_11.5 & $52^{\prime \prime}$ & 0.085 & 0.087 & 10.0 \\
HR 5986 & P_25 & $79^{\prime \prime}$ & 0.667 & 0.588 & 12.3 \\
HR 7310 & P_60 & $180^{\prime \prime}$ & 0.667 & 0.574 & 7.0 \\
HR 7310 & C_100 & - & 0.229 & 0.250 & 10.6 \\
HR 6705 & C_160 & - & 0.672 & 0.631 & 7.2 \\
\hline NGC 6543 & P_11.5 & $52^{\prime \prime}$ & - & 6.8 & 5.9 \\
NGC 6543 & P_25 & $79^{\prime \prime}$ & - & 107 & 1.8 \\
NGC 6543 & P_60 & $180^{\prime \prime}$ & - & 144 & 6.6 \\
NGC 6543 & C_100 & - & - & 59.5 & 2.0 \\
NGC 6543 & C_160 & - & - & 15.9 & 2.6 \\
\hline
\end{tabular}

uncertainties are around 10 to $20 \%$, but can exceed $30 \%$ under exceptional conditions and at low flux levels. The filters of each subsystem were calibrated relative to each other with accuracies better than $10 \%$.

The use of point sources and standard apertures for each filter for the aperture photometer defines a baseline within the parameter space, which serves as a reference for the calibration of further instrument modes like chopped measurements, multi-aperture measurements and mapping.

Acknowledgements. We would like to thank U. Grözinger for vital support. N. Lu and A. Wehrle are acknowledged for helpful comments on an early version of the manuscript. MC thanks the University of Florida for supporting his contributions to this work through subcontracts (under prime grants NAGW4201 and NAG 5-3343) with VRI and, in later years, through subcontract UF99025 with Berkeley.

\section{References}

Acosta-Pulido, J. A. 1999, IDC technical report http: // www. iso.vilspa.esa.es/users/expl_lib/PHT/ chop_report02.ps.gz

Beichman, C. A., Neugebauer, G., Habing, H. J., Clegg, P. E., \& Chester, T. J. 1988, Infrared astronomical satellite (IRAS) catalogs and atlases, vol. 1: Explanatory supplement

Di Benedetto, G. P. 1998, A\&A, 339, 858

Di Benedetto, G. P. 1995, ApJ, 452, 195

Di Benedetto, G. P. 1993, A\&A, 270, 315

Blackwell, D. E., Petford, A. D., Arribas, S., Haddock, D. J., \& Selby, M. J. 1990, A\&A, 232, 396

Blackwell, D. E., Lynas-Gray, A. E., \& Petford, A. D. 1991, A\&A, 245, 567

Blackwell, D. E., \& Lynas-Grey, A. E. 1998, A\&AS, 129, 505
Van Der Bliek, N. S., Bouchet, P., Habing, H. J. et al. 1992, ISO., Messenger, 70, 28

Blum, J., Hajduk, C., Lemke, D., Salama, A., \& Wolf, J. 1990, Infrared Phys., 30, 93

Bratt, P. R. 1977, Semiconductors and Semimetals, vol. 12, Infrared Det. II, ed. Willardson \& Beer, 39

Cayrel De Strobel, G., Hauck, B., Francois, P., et al. 1992, A\&AS, 95, 273

Cohen, M., Witteborn, F. C., Walker, R. G., Bregman, J. D., \& Wooden, D. H. 1995, AJ, 110, 275

Cohen, M., Witteborn, F. C., Carbon, D. F., et al. 1996, AJ, 112,2274

Cohen, M., Walker, R. G., Carter, B., et al. 1999, AJ, 117, 1864

Cohen, M., Walker, R. G., Jayaraman, S., Barker, E., \& Price, S. D. 2001, AJ, 121, 1180

Conrath, B., Flasar, F. M., Hanel, R., et al. 1989, Science, 246, 1454

Cornwall, L. 1997, Report on FCS straylight investigation, IDT internal report

Dierickx, B., Cos, S., Vermeiren, J., et al. 1989, Infrared Technology XV, SPIE vol. 1157, 24

Engelke, C. W. 1992, AJ, 104, 1248

Fouks, B. I., \& Schubert, J. 1995, Proc. SPIE vol. 2475, 487

Gabriel, C., Acosta-Pulido, J., Heinrichsen, I., Morris, H., \& Tai, W.-M. 1997, Proc. of the ADASS VI Conference, ed. G. Hunt, \& H. E. Payne, 108

Golay, M. 1974, Introduction to astronomical photometry (Dordrecht, D. Reidel Publishing Co.) (Astrophysics and Space Science Library, vol. 41), 375, 41

Griffin, M. J., \& Orton, G. S. 1993, Icarus, 105, 537

Grözinger, U., Kirches, S., Lemke, D., et al. 1993, Proc. Photon Detectors for Space Instr., ESA SP-356, 32

Hammersley, P. L., Jourdain De Muizon, M., Kessler, M. F., et al. 1998, A\&AS, 128, 207

Hanel, R., Conrath, B., Flasar, F. M., et al. 1986, Science, 233, 70

Hildebrand, R. H., Loewenstein, R. F., Harper, D. A., Orton, G. S., et al. 1985, Icarus, 64, 64

Huth, S., \& Schulz, B. 1998, ASP Conf. Ser. 145, Astronomical Data Analysis Software and Systems VII, 7, 212

Kessler, M. F., Steinz, J. A., Anderegg, M. E., et al. 1996, A\&A, 315, L27

Klaas, U., Krüger, H., Heinrichsen, I., Heske, A., \& Laureijs, R. (ed.) 1994, ISOPHOT Observer's Manual, version 3.1

Klaas, U., Acosta-Pulido, J. A., Ábrahám, P., et al. 1997, Conf. Proc. ISO to the Peaks ESA SP-419

Kurucz, R. L. 1993, data on magnetic tape

Laureijs, R. J., Klaas, U., Richards, P. J., \& Schulz, B. 1998, ISOPHOT Data Users Manual, version 4.0, SAI/95-220/Dc

Laureijs, R. J., Klaas, U., Richards, P. J., Schulz, B., \& Ábrahám, P. 2001, ISO Handbook, vol. V: PHT-The Imaging Photo-Polarimeter, version 1.2

Lebofsky, L. A. \& Spencer, J. R. 1989, Asteroids II, 128

Lemke, D., Klaas, U., Abolins, J., et al. 1996, A\&A, 315, L64 Lemke, D., Klaas, Ábrahám, P., et al. 1998, SPIE Infrared Astronomical Instrumentation Conference, SPIE 3354-46

Lindal, G. 1992, AJ, 103, 967

Lindal, G. F., Lyons, J. R., Sweetnam, D. N., et al. 1987, J. Geophys. Res., 92, 1, 4987-15001

Mill, J., O'Neil, R. R., Price, S. D., et al. 1994, AIAA, 31, 900

Müller, T., \& Lagerros, J. 1998, A\&A, 338, 340

Müller, T., \& Lagerros, J. 2001, A\&A, submitted 
Müller, T., \& Lagerros, J. 2001, Proc. The Calibration Legacy of the ISO Mission, VILSPA, Spain, Feb. 2001, ESA $\mathrm{SP}-481$, in press

Jourdain de Muizon, M. J.-D., \& Habing, H. 1992, Les Houches Series: Infrared Astronomy with ISO, ed. Th. Encrenaz, \& M. F. Kessler, 129

Okumura, K. 2000, Proc. of ISO Beyond Point Sources: Studies of Extended Infrared Emission, Madrid, Spain, 14-17 Sep. 1999 (ESA SP-455)

Orton, G. S., Griffin, M. J., Ade, P. A. R., Nolt, I. G., \& Radostitz, J. V. 1986, Icarus, 67, 289

Schubert, J. 1993, MPIA internal report, Filter-Transmissions and Detector Responses in ISOPHOT, FM

Schubert, J. 1996, Ph.D. Thesis, Ruprecht Karls Universität, Heidelberg
Sclar, N. 1984, Prog. Quant. Electr., vol. 9, 149

Schulz, B. 1993, Ph.D. Thesis, Ruprecht Karls Universität, Heidelberg

Schulz, B., Huth, S., Kinkel, U., et al. 1999, ESA SP-427

Schulz, B. 2001, Proc. of the Conf. The Calibration Legacy of the ISO Mission, ESA SP-481, in press

Tropf, W. J., \& Thomas, M. E. 1998, Aluminum Oxide $\left(\mathrm{Al}_{2} \mathrm{O}_{3}\right)$ Revisited in: Handbook of Optical Constants of Solids III, Ac. Press, ISBN 0-12-544423-0, 653

Wolf, J., Gabriel, C., Grözinger, U., et al. 1994, Opt. Eng., 33, 26, also publ. in Proc. SPIE, 2019, 82

Wright, E. L. 1976, ApJ, 210, 250

Wright, E. L., \& Odenwald, S. 1980, Bull. A. Astron. Soc., 12, 456 